\title{
DIFFRACTION-LIMITED IMAGING WITH GROUND-BASED OPTICAL TELESCOPES
}

\author{
A. C. S. Readhead, T. S. Nakajima, T. J. Pearson, G. Neugebauer, J. B. Oke, and W. L. W. Sargent \\ Palomar Observatory and Owens Valley Radio Observatory, California Institute of Technology, Pasadena, California 91125 \\ Received 15 July 1987; revised 29 December 1987
}

\begin{abstract}
The construction of diffraction-limited images with ground-based optical telescopes by means of closure-phase observations is explored. The effect of redundant baselines on closure-phase observations is analyzed, and it is shown that for bright objects a nonredundant-mask approach is superior to the use of the full aperture. Observations of closure phase with a nonredundant mask on the $200 \mathrm{in}$. Hale Telescope are presented, and compared with the predictions of atmospheric seeing theory. It is demonstrated that fairly complex images can be constructed from closure-phase observations alone.
\end{abstract}

\section{INTRODUCTION}

Atmospheric propagation effects limit the resolution of ground-based optical observations to 0.15 arcsec under even the most favorable conditions. Under more normal circumstances, the seeing limitation for most optical observations is in the range 1-2 arcsec. A number of areas of fundamental importance to astronomy would benefit greatly from much higher-resolution optical observations. It is therefore very important to explore possible means of overcoming the effects of the atmosphere. One very promising approach is that which has been used by radio astronomers, particularly in Very Long Baseline Interferometry (VLBI), to make images from data that are badly corrupted by propagation effects.

In VLBI, the observed complex visibility function is corrupted by propagation effects, instrumental effects, and uncertainties in the geometry. In addition, the sampling in spatial frequency (the $(u, v)$ plane) is necessarily incomplete and irregular. Nevertheless, images with the full resolution of the telescope array are obtained routinely. In many cases, the quality of these images is limited primarily by thermal receiver noise, and dynamic ranges of over 1000 can be achieved.

The corruption of the phase of the visibility function is always severe in VLBI: Random phase variations $\gg 1$ radian between elements of the array result from propagation effects in the atmosphere, the ionosphere, the interplanetary medium, and the interstellar medium, in addition to instrumental and geometric problems. The amplitude is usually less badly corrupted and can often be calibrated to within about $10 \%$, but there are cases for which the amplitudes are incorrect by factors of $\sim 30$ or more and even in these cases images can be constructed with a dynamic range of $>100$.

The construction of these images requires the determination of the visibility amplitudes and phases which would have been obtained with a coherent telescope array; i.e., it entails the determination to high precision of the object visibility amplitudes and phases. The complex visibility of the object is derived from the observed complex visibility by the explicit or implicit use of the "closure phase" and "closure amplitude" (Rogers et al. 1974; Readhead and Wilkinson 1978; Readhead et al. 1980; Schwab 1980; Cornwell and Wilkinson 1981) and by the use of the constraints that the brightness distribution must be positive and of finite extent.

As was pointed out by Readhead et al. (1980), these methods are not limited to radio frequencies, but can be applied to any system that can produce detectable interference fringes in a time that is short compared with that in which the measured object visibility changes due to the rotation of the instrument relative to the object. They can therefore be applied directly to ground-based optical interferometry in order to determine both the phase and the amplitude of the object visibility, and hence to construct high-dynamicrange, diffraction-limited images. The major difference in the optical case is that the thermal-receiver noise, which is the ultimate limitation at radio frequencies, is replaced by dark current, readout noise, and photon noise. In principle, it should be possible to construct optical images that are limited primarily by these factors.

The potential importance of closure-phase measurements in optical interferometry was first recognized by Rogstad (1968). Rhodes and Goodman (1973) and Brown (1978) discussed the use of pupil-plane masks with large telescopes for measuring the closure phase. These prescient treatments differ from the practice in VLBI in that they derive the visibility phases from the closure phases stepwise from the smallest to the largest aperture spacings, which introduces errors which increase in proportion to the number of steps. Clearly, these approaches assume full aperture coverage or the measurement of a complete set of Fourier components. This assumption, which is often made in papers on optical interferometry, severely limits the imaging potential of these methods.

The new radio imaging methods, which have been so successful in VLBI considering the sparse $(u, v)$ coverage, rely on the determination of the correct object complex visibilities in both sampled and unsampled regions of the $(u, v)$ plane. This is achieved by using the observed closure phases, together with the positivity and finite source extent, as constraints on the brightness distribution. These powerful nonlinear imaging methods, which were developed in radio astronomy to compensate for both propagation effects and sparse $(u, v)$ coverage, have not been fully exploited in the solution of this problem at optical wavelengths.

In most cases in radio astronomy, the signal-to-noise ratio (SNR) of the observed complex visibility in a coherent integration time is much greater than one. Thus the closure phases can be determined for each coherent integration segment and then incoherently averaged with very little loss in sensitivity compared with coherent averaging. However, there are situations in VLBI in which this is not the case and the signal-to-noise ratio in each coherent segment is less than one. The best examples are millimeter VLBI and low-frequency VLBI. In such cases a significant loss of sensitivity would result from the standard scalar averaging of the closure phases, but this loss is easily avoided by vector averaging of the bispectra (see, for example, Peebles 1980) of visi- 
bilities. This method has been used in analyzing millimeter VLBI observations for which the signal-to-noise ratio on one baseline is significantly weaker than on the two other baselines, forming a closed triangle (Rogers et al. 1984). The same considerations apply in optical interferometry, and, in an independent development to that in radio astronomy, Weigelt and Wirnitzer (1983) and Lohmann et al. (1983) extended the technique of speckle holography (Liu and Lohmann 1972; Weigelt 1977; Weigelt 1978) to objects of arbitrary structure in which there is no unresolved component to use as a phase reference.

Recently, Hofmann and Weigelt (1986) and Haniff et al. (1987) have succeeded in constructing optical images based on the use of closure phases and visibility amplitudes. The methods that these two groups have used are quite distinct, but what sets both of them apart from other attempts at optical-image construction is the use of closure phases. Since the closure phase is the basic new piece of information that is used in both of these methods, we will avoid the misleading appellations "bispectrum" and "closure phase" which are sometimes used to distinguish between these two approaches. The real distinction is between the use of 'fully filled apertures' and 'nonredundant masks.' Since they rely on closure phase, both of these techniques can make use of the experience gained from radio interferometry. Once the closure phase has been measured, in conjunction with the visibility amplitude, the image can be constructed using the standard VLBI techniques, as discussed in Sec. V.

The first successful extraction of closure phases from optical observations was made with a filled aperture by the bispectrum technique by Lohmann et al. (1983). This method was recently used to construct the diffraction-limited optical image of the four 11-12 mag stars comprising the central object HD 97950 AB in NGC 3603 (Hofmann and Weigelt 1986).

Baldwin et al. (1986) used a nonredundant aperture mask to derive closure phases from optical observations. Their method is a direct analog of the VLBI situation, apart from the fact that the fringes are superposed on one detector. They made a simple image, or hybrid map, of the $4.5+5.5 \mathrm{mag}$ double star $\phi$ And with a separation of $0.45 \operatorname{arcsec}$ (Haniff $e t$ al. 1987).

These two results are significant since they are the first optical images in which the closure phases have been used. Thus far, no one has made use of the closure amplitude in the construction of an optical image.

In this paper, we discuss the closure phase in optical interferometry and the application of these methods to observations on the 200 in. Hale Telescope. We are engaged in a program of optical interferometry which is aimed at exploiting fully the potential for diffraction-limited imaging with large ground-based optical telescopes. The scientific motivations in stellar astronomy and the study of active galactic nuclei are well known. Our immediate technical objectives are to determine the optimum observing strategy under various conditions of seeing, to adapt the image-construction techniques that have been so successful in radio astronomy to the optical case, and to determine the sensitivity of these methods with ground-based optical telescopes.

We are further motivated to apply radio techniques to optical interferometry by the following considerations:

(1) A number of new high-altitude observatories have seeing considerably better than 1 arcsec for a substantial fraction of the time. The consequent larger lateral coherence lengths and longer coherence times will significantly augment the potential of these methods.

(2) The advent of high-quantum-efficiency photoncounting devices vastly increases the sensitivity of this method, which raises the possibility of making diffraction-limited images of objects as faint as $\sim 15$ mag under good seeing conditions.

(3) Recent experience in radio astronomy has shown that in some circumstances images can be constructed based on phase information alone, and this opens up the possibility of making images from the closure phases alone in situations where even the closure amplitudes are corrupted (see Sec. V).

(4) In the nonredundant-mask technique, the phase differences along different atmospheric paths are determined explicitly. Thus this method is ideal for measuring and characterizing the effects of the atmosphere under different seeing conditions, for it yields directly the spectrum of atmospheric irregularities and the phase and amplitude structure functions. A detailed knowledge of these quantities will be invaluable for optimizing the construction of ground-based optical interferometers (e.g., Roddier and Roddier 1987).

The layout of this paper is as follows: Sec. II is a discussion of the problems that affect ground-based optical imaging of astronomical objects, and in Sec. III we discuss methods for overcoming atmospheric effects, with emphasis on the use of the closure phase. In Sec. IV we describe observations made on the $200 \mathrm{in}$. telescope in which we measured the closure phase of a bright unresolved star ( $\alpha$ Lyrae), and we compare these with results of simulations assuming that the refractive index fluctuations have a Kolmogorov power spectrum with an index of 11/3 (Tatarski 1967). Our observations show that the closure phase can be measured over intervals significantly longer than the atmospheric coherence time (at the cost of introducing a new component of noise-see Sec. III), or using apertures significantly larger than the lateral coherence length and using fractional bandwidths as large as 0.15 . In Sec. V we discuss the application of nonlinear image-construction methods in optical astronomy, some limitations of these methods in observations of strong sources, and the difficulties associated with visibility-amplitude measurements; and we give some examples of blind tests of image construction using closure phases. An Appendix describes simulations of the effects of the atmosphere on visibility amplitude, visibility phase, and closure phase for the case of a Kolmogorov spectrum of refractive-index inhomogeneities.

In this paper, we consider only the case of interferometric observations of bright objects which are not limited by photon noise. The effects of photon noise and the sensitivity limits of this technique will be discussed elsewhere.

\section{DIFFICULTIES IN OPTICAL IMAGING WITH GROUND- BASED TELESCOPES}

The construction of diffraction-limited images by conventional interferometric techniques requires the accurate calibration of both the amplitude and phase of the complex visibility function. In other words, it requires an accurate determination of the complex gain of each antenna or aperture. In a well-designed optical system with a fully filled aperture, in the absence of atmospheric effects, the correct relative amplitudes and phases are automatically preserved and this produces a diffraction-limited image directly.

In practice, it is not necessary to have complete, well-calibrated visibility information to make a good image since the 
positivity constraint and the finite extent of the object, which are often not used explicitly in conventional image construction, place strong constraints on the possible values of the visibility function in both sampled and unsampled regions of the $(u, v)$ plane (Pearson and Readhead 1984; Readhead 1988 , and see Sec. V). For example, in VLBI at high frequencies, it is often not possible to calibrate either the amplitudes or the phases, but nevertheless it has been shown that it is possible to construct images from completely uncalibrated data (Readhead et al. 1980) by a method that depends only on having interference fringes that are detectable in a time that is shorter than the time in which the object visibility changes significantly due to Earth rotation, provided that there are no large systematic errors in the closure phases and closure amplitudes.

In both the optical and radio cases, the phases are corrupted by propagation effects, instrumental effects, and uncertainties of the geometry of the imaging system. Propagation through media with random refractive-index variations introduces random changes in phase. Detector noise introduces a random noise component into the observed phase. Uncertainties in the exact position of different parts of the telescope or array introduce systematic errors into the phase measurements. These effects cancel out in the closure phase, which is why it is such a good observable.

Significant amplitude variations (scintillation) can be introduced by propagation through a medium with random refractive-index variations. A variety of instrumental effects can also reduce the amplitude at optical wavelengths; for example, vibrations, misfocusing, finite pixel size, and finite bandwidth. In general, these effects depend on baseline length and do not, therefore, cancel out in the closure amplitude.

There are four important differences between the optical and radio situations which should be borne in mind:

(1) The fundamental noise limit in optical observations is Poisson photon noise. In radio astronomy, it is Gaussian receiver noise.

(2) At optical wavelengths, the atmospheric coherence time $\tau_{\text {coh }}$ in which the rms phase fluctuation at a stationary point is 1 radian, and the lateral coherence length $r_{\text {coh }}$ over which the instantaneous rms phase deviation is 1 radian, are much smaller than at radio wavelengths, and a large number $\left(\geqslant 10^{3}\right)$ of frames are needed to determine closure phases to within a few degrees.

(3) There is generally a single detector array in the optical case, and all of the fringes are superposed.

(4) The $(u, v)$ coverage at optical wavelengths is generally much better than at radio wavelengths.

\section{SOLUTIONS TO THE SEEING PROBLEM}

The problems of ground-based optical interferometry and the methods employed to circumvent them have been reviewed by C. Roddier (1981), Woolf (1982), and Coulman (1985). There have been a number of successful attempts to overcome the resolution limit imposed by the atmosphere by using different interferometric techniques (McAlister 1985): Michelson interferometry (Michelson 1920), intensity interferometry (Hanbury Brown et al. 1970), and speckle interferometry (Labeyrie 1970). Optical fringes from astronomical objects have been observed by a number of workers since the pioneering observations of Michelson and Pease (1921). Currie, Knapp, and Liewer (1974) used a mask on the 200 in. telescope to make amplitude-interfero- metry measurements of stars. More recently, Shao and Staelin (1980) and Shao et al. (1987) have tracked fringes with a Michelson interferometer.

In general, these methods have not yielded true images of objects because the phase information is either lost or discarded. The construction of images without phase information is difficult, but is possible in some circumstances (Baldwin and Warner 1976; Fienup 1978; Fienup 1984 and references cited therein). Without phase information there always remains a $180^{\circ}$ ambiguity in the orientation of the image. While in many cases this does not seriously hinder interpretation, there are some important applications for which this information is crucial, for example, in high-resolution radio observations of the jets in active galactic nuclei.

In optical interferometry it is extremely difficult to calibrate the amplitudes to within a few percent, as required for making high-dynamic-range images. Thus, over the last dozen years various methods for extracting some phase information from the observations have been suggested (e.g., Knox and Thompson 1974). These methods have generally been applied to speckle observations on large apertures. These developments have been important for the attention that they have focused on the extraction of phase information from the observations, and they provide a partial solution of the imaging problem. However, they generally rely on the stepwise extraction of phase information across the $(u, v)$ plane, and this propagates errors which accumulate as the baseline length is increased. A more powerful approach is the use of the closure phase.

\section{a) The Closure Phase and Closure Amplitude}

We concentrate, to begin with, on the case of Michelson interferometry with a nonredundant mask (Rhodes and Goodman 1973; Baldwin et al. 1986), since the closure phase is easiest to understand in this application. In Michelson interferometry with only two apertures, the randomphase variations due to the atmosphere cannot be calibrated and the fringe phase cannot be measured. However, if three apertures are used, one gets three superimposed fringe patterns, and provided that the aperture spacings (i.e., the baseline lengths or orientations) are different, it is possible to identify the three patterns with the three baselines, and to calculate the closure phase (Jennison 1953, 1958; Rogstad 1968). This extension to three or more apertures is trivial when the apertures are obtained by placing a mask in the light patch of a large telescope (Currie et al. 1974).

The closure phase is a good observable which is unaffected by propagation effects. Indeed, at high photon-count rates it is not affected by any errors, apart from detector noise, that can be attributed to a single telescope or elemental area on a telescope mirror. It is only affected by sources of noise that depend on baseline length, by the source structure, and, if a single detector is used, by redundancy (see Sec. III $b$ ). In Fig. 1 we show schematically that propagation effects (and phase errors associated with individual elements) do not affect the closure phase.

If a fourth aperture is added, then in addition to closure phase one can measure closure amplitude, and it is possible to make reliable images from completely uncalibrated data (Readhead et al. 1980). Note that a four-hole mask provides between three and four independent closure phases, depending on the signal-to-noise ratio, and between two and three independent closure amplitudes (see Sec. III $d 1$ ).

In the following three sections we show that measure- 

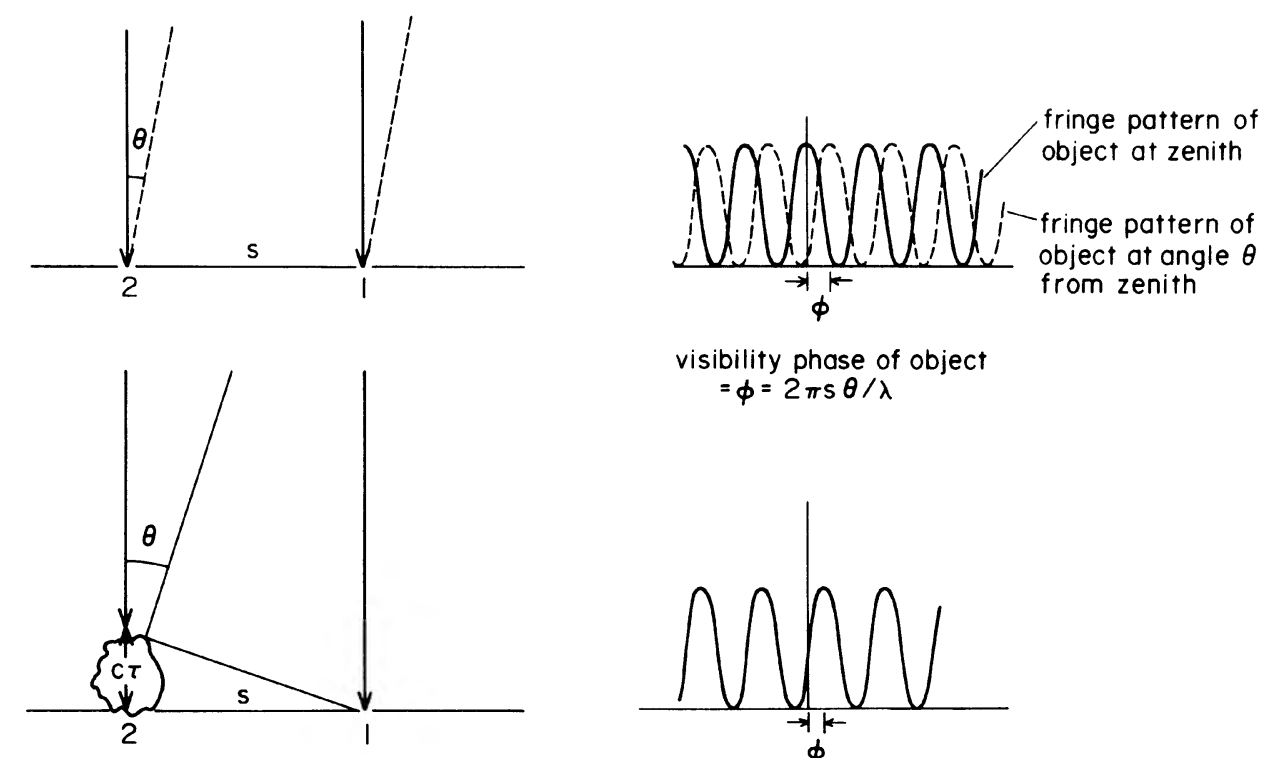

visibility phase of object

$=\phi=2 \pi s \theta / \lambda$

$\theta \cong c \tau / s$

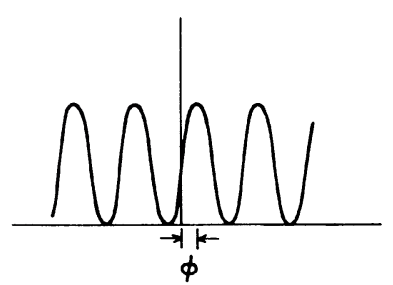

visibility phase error introduced by atmosphere for object at zenith $\phi=2 \pi c \tau / \lambda$

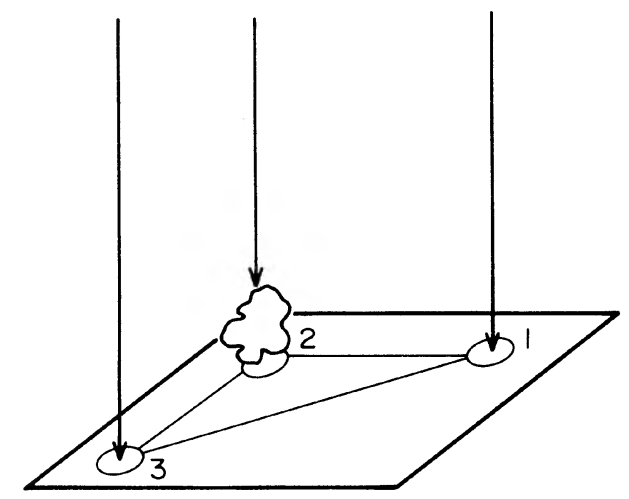

Closure phase $=\phi_{123} \equiv \phi_{12}+\phi_{23}+\phi_{31}=0$

i.e. no error introduced into closure phase by atmosphere

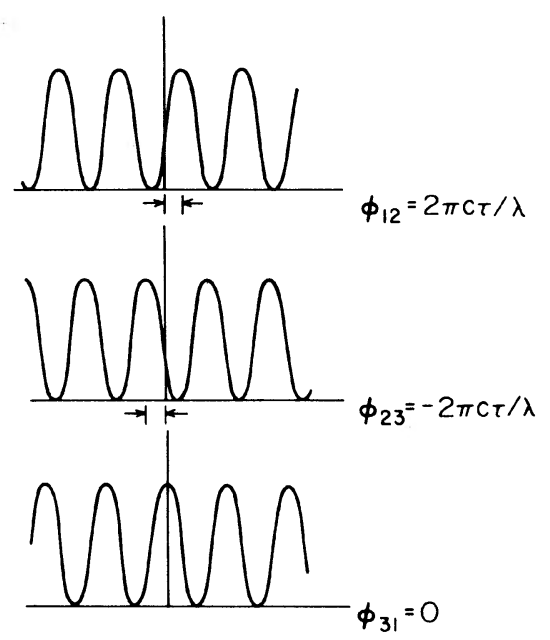

FIG. 1. Illustration of the cancellation of propagation effects in the closure phase. An atmospheric inhomogeneity which introduces a delay $\tau$ in the arrival time of the wavefront at telescope 2 gives rise to equal but opposite shifts in the visibility phase on baselines 12 and 23. Thus these shifts cancel out in the closure phase.

ments of the closure phase can be made using integration times about a factor of 2 longer than $\tau_{\text {coh }}$ without introducing more noise into the closure phase, and that the closure phase can be extracted from observations with integration times $\gg \tau_{\text {coh }}$. We also show that the redundant baselines of a fully filled aperture of diameter $D$ introduce a noise term of rms amplitude $\sim\left(D / r_{\text {coh }}\right)^{3}$ in the closure phase, whereas the amplitude of the closure phase term $\sim\left(D / r_{\text {coh }}\right)^{2}$. Thus the signal-to-noise ratio of the closure phase $\sim\left(r_{\text {coh }} / D\right)$.
The redundancy noise can be eliminated by the use of a nonredundant mask.

\section{b) Nonredundant Masks and Limitations Imposed by the Coherence Time}

It is well known that the closure phase is a good observable when integration times shorter than $\tau_{\text {coh }}$ are used, and that the closure phase enables us to combine short observations 
coherently for periods of arbitrary duration (typically $\sim 12$ $\mathrm{hr}$ in VLBI) by using the object as its own phase reference. It is instructive to consider the effect on the closure phase of increasing the integration time of individual observations beyond $\tau_{\text {coh }}$. In this section we show that, due to the cancellation of some terms in the bispectrum, measurements of the closure phase can be made with integration times about a factor of 2 longer than $\tau_{\text {coh }}$ without increasing the noise, and also that the closure phase can be determined from observations with integration times $\gg \tau_{\text {coh }}$.

We begin by considering the formation of an intensity fringe pattern by interference of light from three elementary areas of the aperture 1,2,3 (see Fig. 1). Let the amplitudes and phases of the intensity patterns corresponding to the three intersecting fringe patterns thus formed be represented by the complex numbers $A_{12} e^{i \phi_{12}}, A_{23} e^{i \phi_{23}}$, and $A_{31} e^{i \phi_{31}}$ on baselines 12,23, and 31, respectively. The closure phase $\phi_{123}$ is given by

$$
\phi_{123}=\phi_{12}+\phi_{23}+\phi_{31} \text {, }
$$

which is the argument of the bispectrum, or triple product, defined by $\mathscr{P}_{123} \equiv A_{12} A_{23} A_{31} e^{i\left(\phi_{12}+\phi_{23}+\phi_{31}\right)}$ (Lohmann et al. 1983; Cornwell 1987). Suppose the atmosphere contributes $\delta_{1}, \delta_{2}, \delta_{3}$ to the phase along the optical path to apertures $1,2,3$, respectively; then the observed phase $\phi_{12}$ is

$$
\phi_{12}=\psi_{12}+\delta_{1}-\delta_{2}+\Delta \phi_{12},
$$

where $\Delta \phi_{12}$ represents the combined effects of detector noise and photon noise, and $\psi_{12}$ is the object visibility phase, i.e., $\psi_{12}=\arg \left[\widetilde{O}_{12}\right]$, where $\widetilde{O}(u, v)$ is the Fourier transform of the object brightness distribution $O(x, y)$. For the present discussion we assume that $\Delta \phi_{12} \ll 1$ radian and we therefore ignore this term. Hence we have the familiar cancellation of atmospheric contributions to the closure phase:

$$
\phi_{12}+\phi_{23}+\phi_{31} \simeq \psi_{12}+\psi_{23}+\psi_{31} \equiv \psi_{123} \text {, say. }
$$

Consider an observation for which the integration time $\tau$ is significantly longer than $\tau_{\text {coh }}$. For simplicity, we assume that the fringe pattern is frozen for intervals $\tau_{0} \sim \tau_{\text {coh }}$, with random phase changes of order 1 radian at each of the three apertures between each interval $\tau_{0}$, and that $\tau=n \tau_{0}$. We will examine the intensity pattern obtained in such a situation. The intensity pattern arising from baseline 12 for an observation of duration $\tau$ is simply the vector sum:

$$
\sum_{j=1}^{n} A_{12_{j}} e^{i\left(\phi_{12_{j}}\right)}
$$

where the subscripts $1,2 \ldots n$ refer to the first, second $\ldots n$th interval of duration $\tau_{0}$. We wish to determine the closure phase in this situation, so we form the bispectrum:

$$
\sum_{j=1}^{n} A_{12_{j}} e^{i\left(\phi_{12_{j}}\right)} \sum_{j=1}^{n} A_{23_{j}} e^{i\left(\phi_{23_{j}}\right)} \sum_{j=1}^{n} A_{31_{j}} e^{i\left(\phi_{31_{j}}\right)} .
$$

There are three types of terms in the above bispectrum:

(1) terms of the form $q_{1}=q_{2}=q_{3}$ :

$$
A_{12_{q}} A_{23_{q}} A_{31_{q}} e^{i\left(\phi_{12_{q}}+\phi_{23 q}+\phi_{31} q_{q}\right.} \text {, }
$$

which give us the closure phase. The phase of these terms is zero for a point source. We will call these 'terms of the first kind.' There are $n$ terms of the first kind.

(2) terms of the form $q_{1}=q_{2} \neq q_{3}$ :

$$
\begin{aligned}
& A_{12_{q}} A_{23_{q}} A_{31_{s}} e^{i\left(\phi_{12_{q}}+\phi_{23_{q}}+\phi_{31_{s}}\right)}, \\
& A_{12_{q}} A_{23_{s}} A_{31_{q}} e^{i\left(\phi_{12_{q}}+\phi_{23_{s}}+\phi_{31_{q}}\right)},
\end{aligned}
$$

and

$$
A_{12_{q}} A_{23_{s}} A_{31_{s}} e^{i\left(\phi_{12_{q}}+\phi_{23_{s}}+\phi_{31_{s}}\right)} \text {; }
$$

which occur in pairs. Note that

$$
\left(\phi_{12_{q}}+\phi_{23_{q}}+\phi_{31_{s}}\right)=\psi_{123}+\left(\delta_{3_{q}}-\delta_{3_{q}}+\delta_{3_{s}}-\delta_{1_{s}}\right)
$$

and

$$
\left(\phi_{12_{s}}+\phi_{23_{s}}+\phi_{31_{q}}\right)=\psi_{123}-\left(\delta_{3_{q}}-\delta_{3_{q}}+\delta_{3_{s}}-\delta_{1_{s}}\right),
$$

and that similarly

$$
\left(\phi_{12_{q}}+\phi_{23_{s}}+\phi_{31_{q}}\right)=\psi_{123}-\left(\delta_{2_{q}}-\delta_{2_{s}}+\delta_{3_{s}}-\delta_{3_{q}}\right)
$$
and

$$
\left(\phi_{12_{s}}+\phi_{23_{q}}+\phi_{31_{s}}\right)=\psi_{123}+\left(\delta_{2_{q}}-\delta_{2_{s}}+\delta_{3_{s}}-\delta_{3_{q}}\right) .
$$

We call these 'terms of the second kind.' There are $3 n(n-1)$ terms of the second kind. If these terms of the second kind are combined in pairs, the resultants either have phase equal to the closure phase (when their phase angles lie in the range $-\pi / 2$ to $\pi / 2$ ), or they differ from the closure phase by $\pi$ (when these angles lie in the range $\pi / 2$ to $3 \pi / 2$ ). Combining $3 n(n-1) / 2$ of these pairs results in a vector of rms length $[3 n(n-1)]^{1 / 2}$ at angle $\phi_{123}$ or $\phi_{123}+\pi$. When the terms of the second kind are added to those of the first kind, they therefore produce no change in the closure phase or they produce a change of $\pi$.

(3) terms of the form $q_{1} \neq q_{2} \neq q_{3}$ :

$$
A_{12_{q}} A_{23_{s}} A_{31_{t}} e^{i\left(\phi_{12_{q}}+\phi_{23_{s}}+\phi_{31_{t}}\right)} \text {. }
$$

These terms have random phases, and therefore they add incoherently. They introduce a noise term into the bispectrum, and corrupt the closure phases of the bispectrum. We call these 'terms of the third kind.' There are $n(n-1)(n$ -2 ) terms of the third kind.

We will assume that all the amplitudes $A_{i j}$ are equal and set these equal to unity; i.e., we assume that the scintillation is negligible and the error in the amplitude measurements is very small and may therefore be ignored in the present discussion. The bispectrum then consists of a coherent (constant phase) term of length $n$, and phase $\phi_{123}$; a term of phase $\phi_{123}$ or $\phi_{123}+\pi$ and rms length $[3 n(n-1)]^{1 / 2}$; and a term of random phase and rms length $[n(n-1)(n-2)]^{1 / 2}$. Note that if only two intervals are considered; i.e., if $n=2$, there are no terms of the third kind, so that the noise in the closure phase is not increased by the longer integration time, but it will be wrong by $\pi 21 \%$ of the time. Thus integration times about a factor of 2 longer than $\tau_{\text {coh }}$ can be used without increasing the noise in closure-phase measurements. However, this is at least in part an artifact of the model, in which we have assumed that the phases change instantaneously and synchronously. The signal-to-noise ratio is $n[n(n$ $-1)(n-2)]^{1 / 2}$, so that for a signal-to-noise ratio of unity, corresponding roughly to an rms errors in the measured closure phase of 1 radian, $n=3.41$.

In order to measure the closure phase, we have to take many exposures and add the bispectra. Since the closure phase is preserved, the terms of the first kind add coherently, while those of the second kind add incoherently and affect the amplitude of the coherent signal. Thus, after summing $N$ exposures we have the situation illustrated in Fig. 2. Note that the amplitude of the fixed phase term is $N n \pm[N 3 n(n-1)]^{1 / 2}$, while the rms amplitude of the incoherent random phase term is $[N n(n-1)(n-2)]^{1 / 2}$. This shows that it is possible to extract closure-phase information from observations that extend to much longer than 


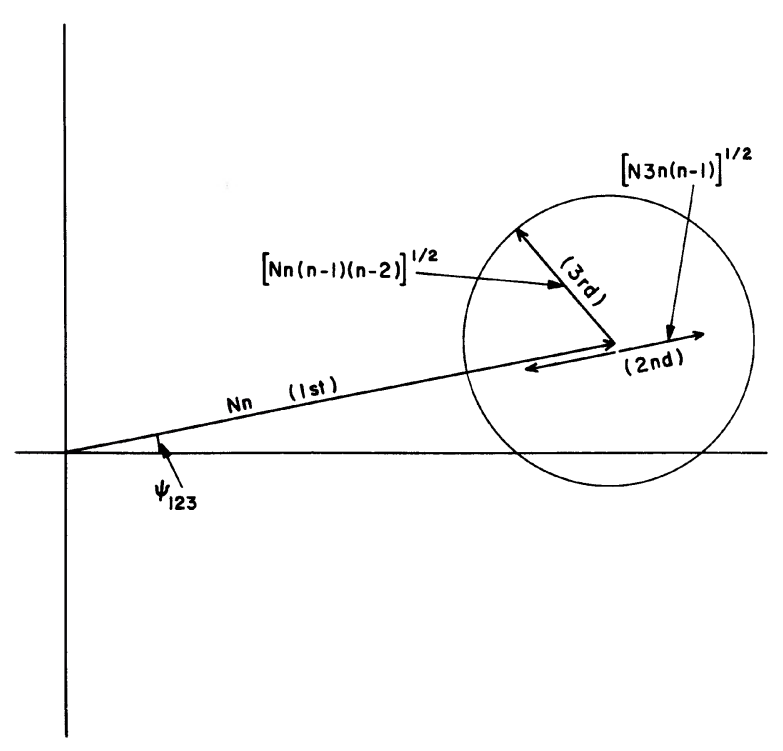

FIG. 2. Effects of redundant baseline noise. The relative magnitudes of the contributions from terms of the first three kinds in the bispectrum are shown. Terms of the first kind add coherently. Terms of the second and third kinds add incoherently. In this example $n=10$; i.e., the integration time is ten times the coherence time, or there are ten identical triplets of apertures contributing instantaneously to the bispectrum; and $N=100$; i.e., the bispectrum has been averaged over one hundred frames.

$\tau_{\text {coh }}$, but that the number of frames needed to reduce the rms error in the closure phase to $1 / x$ radians is $N=x^{2}(n-3+2 / n)$.

\section{c) Fully Filled Apertures}

In speckle observations with a fully filled aperture, the redundancy errors are random and unbiased closure phases can be determined from the bispectrum so that good images can be made provided that enough closure phases are measured (see Sec. V).

This section presents an analysis of the effects of redundant baselines on closure-phase measurements with fully filled apertures for the case of many photons; i.e., ignoring the effects of photon noise. It is shown that the presence of instantaneously redundant baselines gives rise to a noise term very similar to that which is found in integrations that exceed $\tau_{\text {coh }}$, and that for a fully filled aperture of area $D^{2}$ the noise in the closure phase due to the effects of redundant baselines is proportional to $D^{3}$. This 'redundancy noise' term exceeds the photon noise, detector noise, and other sources of noise for observations of bright objects. It is clear from this analysis that the fully filled aperture has some very undesirable properties which can be avoided by using masks (see also Rhodes and Goodman 1973).

The analysis of the previous section can be applied in the spatial domain. Consider the intensity pattern produced by a fully filled aperture. There are many identical triangles of elementary coherence areas in the aperture, and the bispectrum of the complex visibility due to these redundant triangles is again given by Eq. (2). In the case of 'frozen turbulence,' i.e., under the Taylor hypothesis (Taylor 1935), there is no difference between combining the interference fringes from a single triplet of elementary areas over a period of time and combining the fringes from a number of spatially distinct identical triplets at a single instant.

Consider triangles of a particular size, shape and orientation, defined by three vector baselines 12,23 , and 31 . In general, there will be $n$ triangles of this shape, but the bispectra will also contain contributions from baselines that do not form part of triangles of this shape. Suppose that there are $p$ baselines $12, q$ baselines 23 , and $r$ baselines 31 which are not parts of such triangles. Then the bispectrum is

$$
\sum_{j=1}^{n+p} A_{12_{j}} e^{i\left(\phi_{12_{j}}\right)} \times \sum_{j=1}^{n+q} A_{23_{j}} e^{i\left(\phi_{23_{j}}\right)} \times \sum_{j=1}^{n+r} A_{31_{j}} e^{i\left(\phi_{31_{j}}\right)} .
$$

Thus now, in addition to the terms of the first three kinds discussed above, there are terms of the kind

$$
A_{12_{s}} A_{23_{t}} A_{31_{w}} e^{i\left(\phi_{12_{s}}+\phi_{23_{t}}+\phi_{31_{w}}\right)}, \text { etc. }
$$

with one or more of $s, t$, and/or $w>n$, which we shall call 'terms of the fourth kind.' There are $(p+q+r) n^{2}$ $+(p q+q r+r p) n+p q r$ terms of the fourth kind. The incoherent or noise term in the bispectrum thus has an rms amplitude of $\left[n^{3}+(p+q+r-3) n^{2}+(p q+q r+r p\right.$ $+2) n+p q r]^{1 / 2}$, and in order to measure the closure phase to within an rms error of $1 / x$ radians we require $N$ $>x^{2}[n+(p+q+r-3)+(p q+q r+r p+2) / n+p q r /$ $\left.n^{2}\right]$.

The bispectrum approach works with fully filled apertures because the closure phase is preserved by the terms of the first kind. It is only because there are some closed triangles that any information about the phase is recoverable. In the case of a filled aperture, there are triangles for which $n \gg p$ and triangles for which $p \gg n$, etc., so the number of exposures required for a given signal-to-noise ratio can be set by either of these.

We consider a square aperture and assume, for convenience, that the coherent patches are also square (see Fig. 3), and we also assume, to begin with, that the phase distribution across the aperture can be approximated by a uniform grid of $m \times m$ elementary areas of size $r_{\text {coh }}$, each of which has a constant phase, with the value of the phase being selected randomly from the range $-\pi$ to $\pi$. This imposes much more regularity than the real situation, but leads to the correct qualitative results, as is confirmed by computer simulations (Nakajima, in preparation). We also discuss, by way of example, only triangles for which two sides are parallel to the sides of the aperture. In this case, all baselines are included in at least one of the $2 m(m-1)-2$ such triangles, and $r=0$. The number of identical triangles is $n=\left(m-l_{1}\right.$ $+1)\left(m-l_{2}+1\right)$, where $l_{1}, l_{2}$ are the lengths of the sides of the triangle parallel to the sides of the aperture. We will discuss just two examples of triangles in such an aperture. These are marked "a" and " $b$ " in Fig. 3. Then we can calculate the quantities in Table I. Thus, for example, for $m=10$ the signal-to-noise ratio for triangles of both types is 0.10 .

The signal-to-noise ratio for large $m$ is given approximately by $\left[\left(m-l_{1}\right)\left(m-l_{2}\right)+m^{2} /\left(m-l_{1}+1\right)\left(m-l_{2}\right.\right.$ $+1)]^{-1 / 2}$. It is easy to show that this is approximately proportional to $1 / \mathrm{m}$ for most triangles. In fact, only those with $l>0.9 m$ deviate from this dependence by more than $10 \%$ for values of $m>20$. There are very few of these, and so it is a good approximation to assume that the rms noise in the closure phases is proportional to $\mathrm{m}^{3}$. 


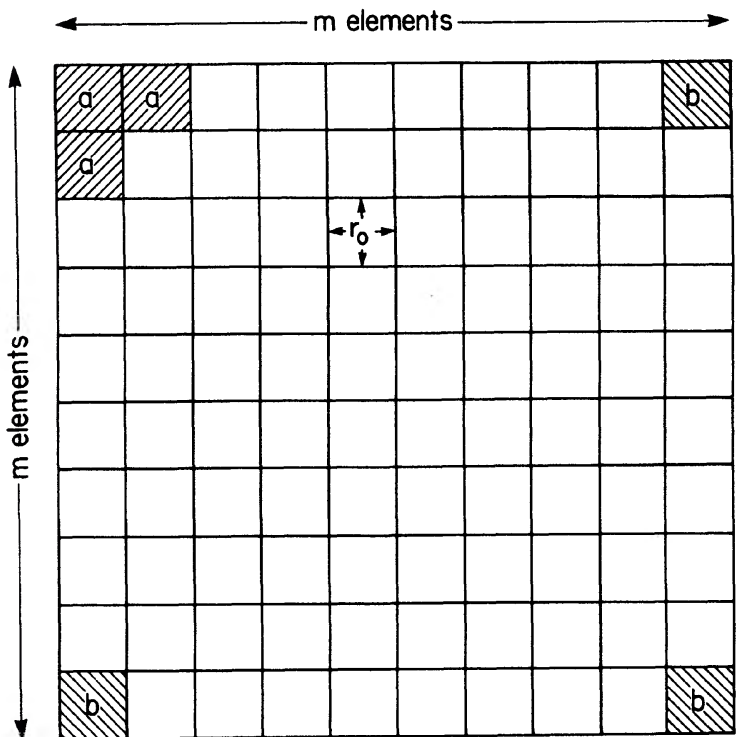

FIG. 3. Coherent-phase patches over a fully filled aperture. It is assumed that each elementary square, of area $r_{\text {coh }}^{2}$, has a constant phase, and that the phases change randomly between patches. This imposes more regularity than obtains in the real situation, but gives results that are qualitatively correct (see the text).

In the real situation we do not have a regular grid but a random distribution in two dimensions. In this case, there are fewer identical triangles, and the probability $\epsilon$ of having triangles of a certain size and shape is a constant per unit aperture area which is determined by the characteristics of the scattering medium. The ratio of the coherent signal to the redundant baseline noise is

$$
\begin{aligned}
& {[\epsilon n+(p+q+r-3)+(p q+q r+r p+2) /} \\
& \left.(\epsilon n)+p q r /(\epsilon n)^{2}\right]^{-1 / 2},
\end{aligned}
$$

for $\epsilon n \geqslant 1$ and 0 for $\epsilon n<1$. If $\epsilon n \gg 1$, the rms noise in the closure phases for large $m$ is therefore still proportional to $m^{3}$.

This analysis shows that the advantage, if it exists, of fully filled apertures over nonredundant masks is not as great as might at first be supposed. We show below that in the case of bright objects, for which photon noise is not a significant problem, the nonredundant mask technique is preferable to the fully filled aperture method. Roddier (1987) has reached a similar conclusion from an analysis of redundant versus nonredundant beam recombination.

\section{d) Comparison of Nonredundant Masks and Fully Filled Apertures}

In this section we compare the nonredundant-mask and fully filled aperture methods. It is shown that for objects brighter than $8 \mathrm{mag}$ the nonredundant-mask method is more sensitive than the fully filled aperture method, and that this is likely true down to much fainter levels. It is by no means clear that the fully filled aperture method is superior even for faint objects, but a complete analysis of the covariance of the bispectrum which takes account of the characteristics of atmospheric noise is required to make an exact determination of the signal-to-noise ratio of a map made by either of these methods. Such an analysis based on both simulations and analytic calculations is in progress (Nakajima, in preparation; Kulkarni and Prasad, in preparation). Pending these detailed calculations, we present here useful first-order estimates based on the results of the previous two sections.

Experience with optical interferometry on the 200 in. telescope shows that we can use apertures of $20 \mathrm{~cm}$ diameter in 1 arcsec seeing conditions. In this case, $m \sim 25$ and so, from the results of the previous section, the signal-to-noise ratio for most closure phases is 0.040 . Thus about 600 independent frames are needed to reduce the rms noise in the closure phase to below 1 radian. This immediately demonstrates a significant disadvantage of the fully filled aperture method, since for bright objects the closure phase observed in each frame with a nonredundant mask is less than 1 radian (see Sec. IV). On the other hand, we show below that more clo-

TABLE I. Redundancy noise for observations with a fully filled aperture.

\begin{tabular}{lcc}
\hline \hline & $\begin{array}{c}\text { Triangles similar } \\
\text { to "a" }\end{array}$ & $\begin{array}{c}\text { Triangles similar } \\
\text { to "b" }\end{array}$ \\
\hline $\begin{array}{l}\text { length of side } l_{1} \\
\text { length of side } l_{2}\end{array}$ & 2 & $m$ \\
$\begin{array}{l}\text { Number }(=n) \\
\text { of similar triangles }\end{array}$ & $(m-1)^{2}$ & 1 \\
$\begin{array}{l}\text { number }(=p) \\
\text { of unmatched, redundant } \\
\text { baselines, } 12 .\end{array}$ & $m-1$ & $m-1$ \\
$\begin{array}{l}\text { number }(=q) \\
\text { of unmatched, redundant } \\
\text { baselines, } 23 .\end{array}$ & $m-1$ & $m-1$ \\
$\begin{array}{l}\text { number }(=r) \\
\text { of unmatched, redundant } \\
\text { baselines, } 31 .\end{array}$ & 0 & 0 \\
$\begin{array}{l}\text { Signal-to-noise ratio } \\
\left\{=\frac{1}{[n+p+q-3+(p q+2) / n]^{1 / 2}}\right\}\end{array}$ & {$\left[(m-1)^{2}+2(m-1)+1-1 /(m-1)^{2}\right]^{-1 / 2}$} & \\
\begin{tabular}{l} 
SNR for large $m$ \\
\hline \hline
\end{tabular} & $\propto 1 / m$ & $\propto 1 / m$ \\
\hline
\end{tabular}


sure phases can be measured with a fully filled aperture than with the nonredundant mask so that it is clear that each of the two methods we are considering has some advantages over the other. The tradeoffs between these are discussed below.

\section{1) Independence of bispectra}

Consider a nonredundant mask consisting of $\eta_{a}$ apertures, and a fully filled square aperture of area $D^{2}$. For simplicity, we again assume that there are $m^{2}$ coherent patches of area $r_{\text {coh }}^{2}$ in the fully filled aperture, so that $m \sim\left(D / r_{\text {coh }}\right)$.

We define a complete set of distinct bispectra by the set of closed triangles of baselines that cannot be reproduced by simple translation or rotation by $180^{\circ}$ of other triangles in this set. (Note that triangles rotated by $180^{\circ}$ have the same three baselines and orientations, and the phase simply changes sign). The number $N_{\mathrm{db}}$ of distinct bispectra thus defined is given in Table II.

We define a complete set of statistically independent bispectra by a set of closed triangles chosen such that each member of the set contains one (and only one) baseline which is not contained in any other triangle that is a member of the set. The number $N_{\text {si }}$ of statistically independent bispectra thus defined is given in Table II.

In the following discussion, we show that all of the information available in observations of bright objects with a nonredundant mask is contained in the $N_{\mathrm{si}}$ statistically independent bispectra, but that, in observations with a fully filled aperture and in observations of faint objects, there is more information than is contained in the $N_{\mathrm{si}}$ statistically independent terms. This comes about because the noise in the $N_{\text {db }}$ distinct bispectra is partially correlated and the degree of correlation is a function of the signal-to-noise ratio.

We begin by considering the meaurement of bispectra with a nonredundant mask. We denote the complex visibility

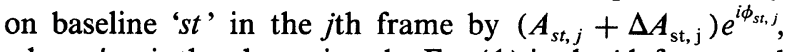
where $\phi_{s t, j}$ is the phase given by Eq. (1) in the $j$ th frame and $\Delta A_{s t, j}$ is the amplitude error due to all sources of noise in the $j$ th frame. We denote actual errors by $\Delta$, rms errors by $\sigma$ and atmospheric delay terms by $\delta$. Note that $\delta_{s}, \delta_{t}, \Delta \phi_{s t}$, and $\Delta A_{s t}$ change from frame to frame but $\psi_{s t}$ does not. The bispectrum is then given by

$$
\begin{aligned}
\mathscr{P}_{p q r}= & \frac{e^{i \psi_{p q r}}}{N} \sum_{j=1}^{N}\left(A_{p q, j}+\Delta A_{p q, j}\right) \\
& \times\left(A_{q r, j}+\Delta A_{q r, j}\right)\left(A_{r p, j}+\Delta A_{r p, j}\right) \\
& \times \exp \left(i\left(\Delta \phi_{p q, j}+\Delta \phi_{q r, j}+\Delta \phi_{r p, j}\right)\right),
\end{aligned}
$$

since the atmospheric-delay terms cancel. In Eq. (3) $\psi_{p q r}$ is the object closure phase on the triangle 'pqr.' Note that a similar expression applies for the fully filled aperture case, but that in this case the noise terms $\Delta \phi_{s t}$ include redundancy noise as well as detector and photon noise. As shown in Sec. III $c$, the redundancy noise in the closure phase is generally much greater than 1 radian.

In the general case when $\Delta A_{s t}$ and $\Delta \phi_{s t}$ can be large, it is not possible to derive a bispectrum $\mathscr{P}_{p q r}$ from three other bispectra which involve the baselines $p q, q r$, and $r p$ because the averaging of the bispectrum is a vector addition and the length of the vector changes from frame to frame. This is a fundamental difference between averaging the bispectrum to determine the closure phases and scalar averaging of the closure phases.

For bright objects the errors are small, and if $\Delta A_{s t, j} \ll A_{s t}$ and $\Delta \phi_{s t, j} \ll 1$, then Eq. (3) may be written

$$
\begin{aligned}
\mathscr{P}_{p q r} \simeq & A_{p q} A_{q r} A_{r p} e^{i \psi_{p q r}} \\
& \times \sum_{j=1}^{N}\left(1+i\left(\Delta \phi_{p q, j}+\Delta \phi_{q r, j}+\Delta \phi_{r p, j}\right)\right) / N \\
= & A_{p q} A_{q r} A_{r p} e^{i \psi_{p q r}} \\
& \times\left(1+i\left(\overline{\Delta \phi}_{p q}+\overline{\Delta \phi}_{q r}+\overline{\Delta \phi}_{r p}\right)\right)
\end{aligned}
$$

where

$$
\overline{\Delta \phi}_{s t}=\sum_{j=1}^{N} \Delta \phi_{s t, j} / N .
$$

Thus, with a nonredundant mask, when the signal-to-

\begin{tabular}{|c|c|c|c|}
\hline & & Nonredundant mask & Full aperture \\
\hline $\begin{array}{l}\text { Number of independent } \\
\text { baselines }\left(N_{\mathrm{b}}\right)\end{array}$ & & $\eta_{a}\left(\eta_{a}-1\right) / 2$ & $2 m(m-1)$ \\
\hline Number of distinct & & $\eta_{a}\left(\eta_{a}-1\right)\left(\eta_{a}-2\right)$ & $3 m^{4}-10 m^{3}+17 m^{2}-18 m+8$ \\
\hline bispectra $\left(N_{\mathrm{db}}\right)$ & & 6 & 4 \\
\hline For large $\eta_{a}, m$ & $N_{\mathrm{db}} \sim$ & $\eta_{a}^{3} / 6$ & $3 m^{4} / 4$ \\
\hline $\begin{array}{l}\text { For circular aperture } \\
\text { and large } m \\
\text { ( } m=\text { diameter) }\end{array}$ & $N_{\mathrm{db}} \sim$ & & $\pi^{2} m^{4} / 32$ \\
\hline $\begin{array}{l}\text { Number of stastically } \\
\text { independent bispectra } \\
\left(N_{\mathrm{si}}\right)\end{array}$ & & $\left(\eta_{a}-1\right)\left(\eta_{a}-2\right) / 2$ & $2 m(m-1)-2$ \\
\hline For large $\eta_{a}, m$ & $N_{\mathrm{si}} \sim$ & $\eta_{a}^{2} / 2$ & $2 m^{2}$ \\
\hline
\end{tabular}
noise ratio in each frame is high, the measured bispectrum reduces to a vector addition in which the length of the vector is approximately constant. The measured bispectrum is now given by the object bispectrum, having length $A_{p q} A_{q r} A_{r p}$ and phase $\psi_{p q r}$ plus a noise term in quadrature. Since $\Delta \phi_{s t, j} \ll 1$ and since $\overline{\Delta \phi}_{s t}$ decreases with increasing $N$, we have $\overline{\Delta \phi}_{\text {st }}$

TABLE II. The numbers of statistically independent and distinct bispectral components for observations with a nonredundant mask and fully filled aperture. 
$\ll 1$ for all $s$ and $t$. Hence the phase of the measured bispec$\operatorname{trum} \phi_{q p r}$ is given by

$$
\begin{aligned}
\phi_{p q r} \simeq & \psi_{p q r}+\overline{\Delta \phi}_{p q}+\overline{\Delta \phi}_{q r}+\overline{\Delta \phi}_{r p}, \\
\text { i.e., } & \\
\phi_{p q r} \simeq & \left(\psi_{p q}+\overline{\Delta \phi}_{p q}\right)+\left(\psi_{q r}+\overline{\Delta \phi}_{q r}\right) \\
& +\left(\psi_{r p}+\overline{\Delta \phi}_{r p}\right) .
\end{aligned}
$$

Thus for observations of bright objects with a nonredundant mask, for which the errors due to photon noise and detector noise are relatively small, the measured phase of the bispectrum averaged over $N$ frames is simply the sum around a closed triangle of baselines, of the three object phases plus the mean phase noise on each baseline. In this case there is no advantage in computing bispectra, and the scalar averaging of closure phases is the preferred approach. This situation obtains in most radio astronomical imaging, and in optical observations of bright objects, as shown below. The number of statistically independent bispectra, and hence the number of statistically independent closure phases, is simply $N_{\text {si }}$ (see Table II).

For the fully filled aperture, on the other hand, $\Delta \phi_{s t} \gg 1$ due to redundancy noise, as shown in Sec. III $c$ and therefore there is more information than is contained in the $N_{\mathrm{si}}$ statistically independent bispectra.

\section{2) Comparison of the two methods}

We now consider observations of a bright object with arbitrary structure, and compare the signal-to-noise ratio on a map of the object made with a fully filled aperture to that on a map made by the nonredundant-mask method. We call this ratio ' $\mathscr{R}$ ', and we denote the crossover magnitude at which the fully filled aperture becomes more sensitive than the nonredundant mask by $m_{\times}$.We assume that, in a single frame, the noise in a bispectrum measurement in the fully filled aperture method is dominated by redundancy noise, which is certainly true for bright enough objects. We denote the rms noise, in a single frame, in the closure phase measured with a nonredundant mask by $\sigma_{c}$, where the subscript ' $c$ ' indicates that this is a closure phase. We denote the signal-to-noise ratio of a closure-phase measurement, determined by averaging the bispectrum over $N$ frames, by $\mathscr{S}_{\mathrm{c}}$. We assume that the signal-to-noise ratio in the visibility amplitudes is not significantly higher than the typical signal-to-noise ratio of the closure phases. Indeed, the following argument applies even to maps made using only closure phases.

From $N$ frames, as shown in the previous two sections, we have, for the fully filled aperture, $\mathscr{S}_{\text {c,FFA }} \sim(N / n)^{1 / 2}$, and, for the nonredundant mask, $\mathscr{S}_{\mathrm{c}, \mathrm{NRM}} \sim N^{1 / 2} / \sigma_{\mathrm{c}}$.

In conventional aperture synthesis, maps are made by linear combinations of the visibilities and we have

$\mathrm{SNR}_{\text {map }} \propto(\mathrm{SNR}$ in visibilities $) \times($ number of baselines) $)^{1 / 2}$.

When closure quantities are used, the maps are made by nonlinear image-construction methods (see Sec. V), but with sufficient care, it is often possible to achieve noise levels that are set by detector noise. Furthermore, as the blind tests of Sec. V show, it is also often possible to achieve noise levels that are set by the detector noise in maps made from closure phases alone; i.e., in these cases

$\mathrm{SNR}_{\text {map }} \propto(\mathrm{SNR}$ in closure phases $) \times($ number of independent closure phases $)^{1 / 2}$.

In the following discussion we therefore assume that the maps made from closure phases alone are limited primarily by random noise, and not by systematic effects introduced by the nonlinear image processing, and that the signal-to-noise ratio is given by the above expression. In this case it is clear that a lower limit to the signal-to-noise ratio in the maps is given by

$$
\mathrm{SNR}_{\text {map }} \propto \mathscr{S}_{\mathrm{c}} N_{\mathrm{si}}^{1 / 2} \text {. }
$$

If the noise in the $N_{\mathrm{db}}$ distinct bispectra were statistically independent, which they are not, we could derive from these a set of $N_{\mathrm{db}}$ statistically independent closure phases, in which case we would have

$$
\mathrm{SNR}_{\text {map }} \propto \mathscr{S}_{\mathrm{c}} N_{\mathrm{db}}^{1 / 2}
$$

Since the $N_{\mathrm{db}}$ distinct bispectra are not statistically independent, Eq. ( $5 \mathrm{~b}$ ) provides an upper limit to the signal-tonoise ratio.

In the following discussion we use these limiting expressions, together with the results of our observations described in Sec. IV, to compare the nonredundant-mask and fully filled aperture methods.

We consider first the case of bright objects. We assume that, for observations with nonredundant masks, provided $\sigma_{A} / A$ and $\sigma_{\mathrm{c}}$ are both $<0.2$, there is little advantage in computing the full set of $N_{\mathrm{db}}$ bispectra. Thus Eq. (5a) is appropriate for nonredundant masks. For observations with a fully filled aperture $\sigma_{\mathrm{c}} \gg 1$ in general, as shown in Sec. III $c$, and therefore there is more information than is contained in the $N_{\text {si }}$ statistically independent bispectra. In this case, the signal-to-noise ratio of the fully filled aperture map lies between the limits given by Eqs. (5a) and ( $5 b$ ).

We derive a lower limit to $m_{\times}$by using Eq. (5a) for the nonredundant mask method and Eq. ( $5 \mathrm{~b}$ ) for the fully filled aperture method. The quantities given in Table II yield $\mathscr{R}=(3 / 2)^{1 / 2} \sigma_{c} m / \eta_{a}$. The case of interest here has $\eta_{a} \sim m \sim n^{1 / 2}$ and hence $\mathscr{R}<1$ for $\sigma_{\mathrm{c}}<(2 / 3)^{1 / 2}$, which does not depend on $m$. This limit is less severe than the limit of $\sigma_{\mathrm{c}}<0.2$.Therefore the nonredundant-mask method is superior to the fully filled aperture method for bright sources. Using the limit of $\sigma_{\mathrm{c}}<0.2$ we estimate, from our observations on the 200 in. telescope, that the nonredundant-mask technique is superior to the fully filled aperture at least down to magnitude 5 in conditions of 1 arcsec seeing. Thus we have $m_{\times}>5$.

At intermediate light levels where the noise in the fully filled aperture method is dominated by redundancy noise, and $\sigma_{\mathrm{c}, \mathrm{NRM}} \sim 1$, it is worthwhile to take the vector average of the bispectra in both techniques.

In this case we may use Eq. ( $5 \mathrm{~b}$ ) for both the nonredundant mask and the fully filled aperture methods to derive a conservative limit on $m_{\times}$. We find, from the quantities given in Table II, $\mathscr{R}=(3 / \sqrt{ } 2) \sigma_{\mathrm{c}} m \eta_{a}^{-3 / 2}$, which, under the same conditions as before, yields $\mathscr{R}<1$ for $\sigma_{\mathrm{c}}$ $<(\sqrt{ } 2 / 3) m^{1 / 2}$. For a large telescope, $m \sim 25$, and by combining this result with the noise levels that we measured on the 200 in. telescope we find $m_{\times}>8$. Note that, because the dependences on $m$ and $\eta_{a}$ do not approximately cancel out, as they did in the previous case, $\mathscr{R}$ decreases as the seeing improves.

This limit on $m_{\times}$is conservative for the following reasons:

(i) The noises of the $N_{\mathrm{db}}$ independent bispectrum terms are not completely uncorrelated and $m_{\times}$will be significantly fainter than 8. For example, if only $N_{\mathrm{si}}$ bispectrum terms are 
used for both the nonredundant-mask and fully filled aperture methods $\mathscr{R}=2 \sigma_{\mathrm{c}} / \eta_{a}$ and hence $\mathscr{R}<1$ for $\sigma_{\mathrm{c}}<m / 2$, which yields $m_{\times}>9.5$.

(ii) These calculations are all based on the smallest triangles and for these the fully filled aperture gives the maximum advantage. For large triangles, the fully filled aperture has significant disadvantages relative to the nonredundant-mask technique, mainly due to the effects of redundancy terms of the fourth kind.

(iii) We have ignored redundancy-noise terms of the fourth kind.

(iv) We have ignored other sources of noise in the fully filled aperture method in comparison with the redundancy noise.

(v) The noise $\overline{\Delta \phi}_{\mathrm{c}}$ depends on both the relative strengths of the fringes on three particular baselines and the background from all points in the aperture not contributing to these baselines. There is thus a component of the noise that is much larger in the case of the full aperture than for the nonredundant mask.

(vi) $m \propto r_{\text {coh }}^{-1}$ so that $m_{\times}$increases as the seeing improves. Thus, the better the seeing the greater is the advantage of using the nonredundant mask.

(vii) For observations of bright objects with a nonredundant mask there are many ways of choosing the independent bispectra. One could, for example, adopt a strategy in which the number of long baselines was minimized; or one in which the number of short baselines was minimized. The optimum strategy depends on the brightness distribution of the object under study. We have neglected some effects which could make the signal-to-noise ratio very low in some cases, for example on long baselines with heavily resolved objects. In other words, the choice of the $N_{\text {si }}$ can be optimized, for example, by selecting those bispectra with large amplitudes so that the highest-signal-to-noise-ratio results are used in the nonredundant-mask method.

(viii) In the simplest nonredundant-mask approach, most of the light incident on the primary mirror is wasted. Some of this could be recovered by using a reflective nonredundant mask so that the light not transmitted could be passed through a second mask. This procedure could be repeated until the reflective losses made it impracticable. In this way it should be possible to increase the sensitivity of the nonredundant-mask method by at least a factor of 3 or 4 .

(ix) An alternative approach which we are exploring is to use masks with some redundancy and impose a 'minimum redundancy' condition which maximizes the number of independent closure phases while restricting the number of redundant baselines.

For these reasons we expect that the nonredundant (or partially redundant) mask technique will prove to be superior to the fully filled aperture method for objects considerably fainter than $8 \mathrm{mag}$. We know that we can compensate very well for nonuniform coverage of the aperture plane, and this is not exploited in the full-aperture speckle approach.

Note that at very low light levels the noise in the singleframe closure phases will not be dominated by redundancy noise because there are so few photons, and therefore the analysis used above does not apply.

It is possible to increase the sensitivity in both the nonredundant-mask method and the fully filled aperture method by using filters over the apertures, and sorting the beams thus produced according to wavelength; for example, by means of dichroic filters.

\section{CLOSURE-PHASE OBSERVATIONS ON THE 200 IN TELESCOPE}

In order to test the feasibility of making closure-phase measurements at optical wavelengths, in August 1985 we carried out a series of observations at the coudé focus of the 200 in. telescope by imaging the $200 \mathrm{in}$. primary mirror onto a mask with appropriate apertures. The light was then focused onto a Texas Instruments CCD detector. The integration time was controlled by means of a shutter. In all of the observations discussed here, a bandwidth of $1000 \AA$ centered on $6500 \AA$ was used.

The experimental setup used in our observations is shown in Fig. 4. The coudé focus of the 200 in. telescope has a focal ratio of 30. The light was focused onto the coudé slit, which was opened up for these observations. The light then passed through a $1200 \mathrm{~mm}$ focal-length lens placed at the focal point beyond the coude slit. The resulting parallel beam was $4 \mathrm{~cm}$ in diameter. The beam then passed through a pupil mask consisting of three holes and onto the coudé collimator, and hence via two flats to the CCD detector. The focal length of the $1200 \mathrm{~mm}$ lens was chosen to provide a convenient scale at the CCD (100 pixels per arcsecond), and it, incidentally, also provided a convenient scale for the maska reduction factor of 127 over the primary mirror. The pupil masks could be exchanged and rotated easily, thus providing a variety of different $(u, v)$ configurations.

\section{COUDE FOCUS OPTICS}

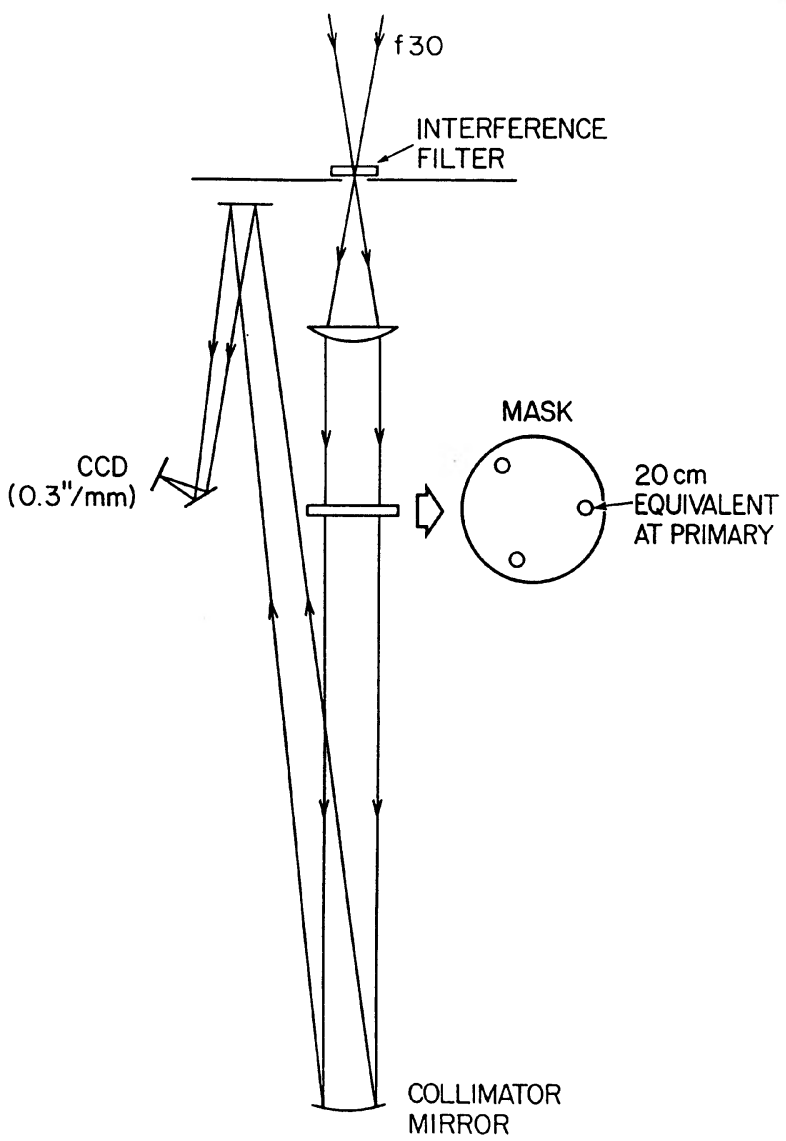

FIG. 4. Setup of the apparatus used in the closure-phase observations on the 200 in. telescope described in the text. 
Observations were made with masks having apertures corresponding to 10,15 , and $20 \mathrm{~cm}$ at the primary. In 1 arcsec seeing, no difficulties were encountered with the 20 $\mathrm{cm}$ mask, indicating that apertures significantly larger than the lateral coherence length $r_{\text {coh }}(\sim 10 \mathrm{~cm}$ for 1 arcsec seeing) can be used.

The interference pattern made with three apertures each of diameter equivalent to $20 \mathrm{~cm}$ at the primary is shown in Fig. 5(a). The amplitudes and phases of the three superimposed sets of fringes can be extracted by taking the complex

(a)

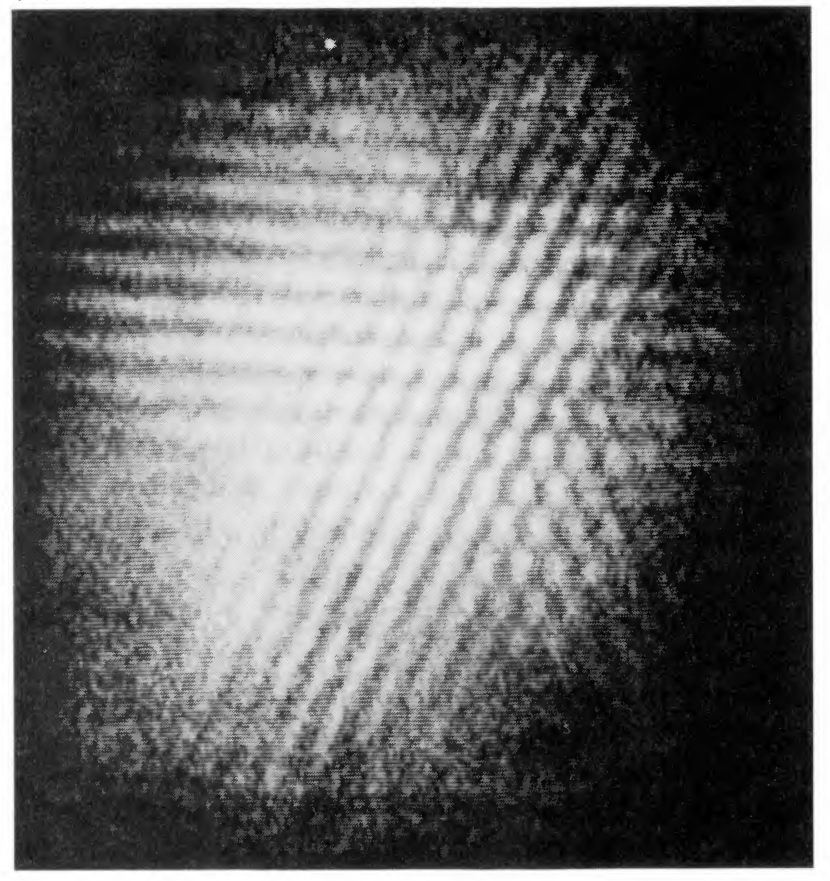

(b)

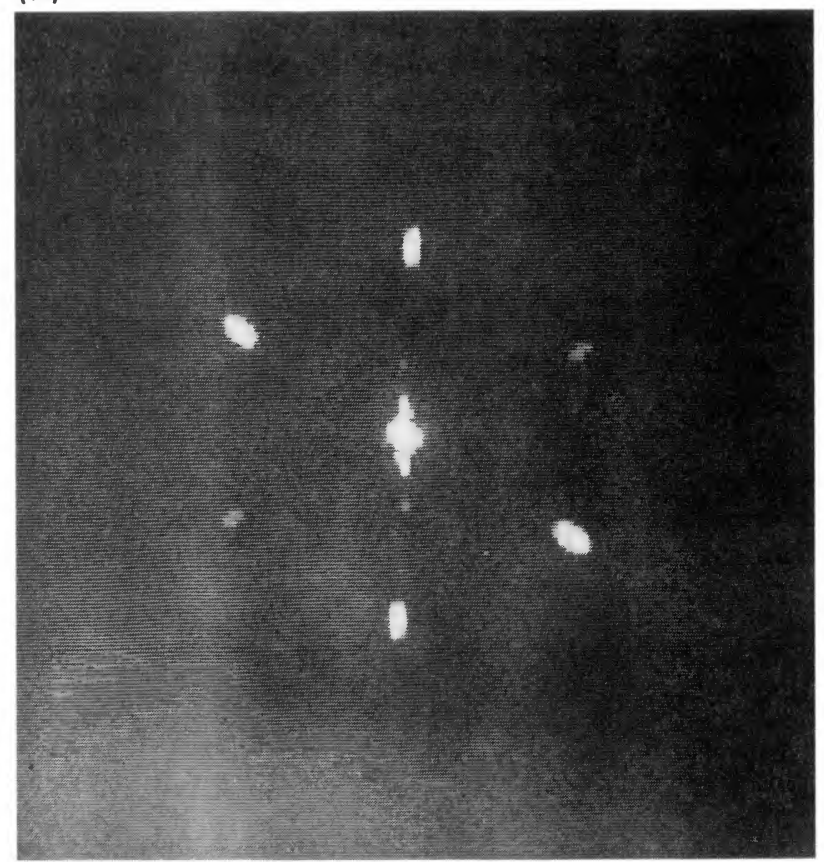

FIG. 5. (a) Interference fringe pattern on Vega made with a three-hole mask. For this image, the integration time was $0.1 \mathrm{~s}$, the bandwidth was $1000 \AA$, and the equivalent diameter at the primary mirror of the holes in the mask was $20 \mathrm{~cm}$. (b) Amplitude of the complex Fourier transform of the image shown in Fig. 5(a).
Fourier transform of this pattern. The amplitude component of the Fourier transform is shown in Fig. 5(b). We see here a bright component at zero spatial frequency, corresponding to the total power in the image, and six fainter components -2 for each pair of apertures, since the Fourier transform of a real function (the pattern, Figure 5(a)) is Hermitian. There are only three independent phases among the six points, the three others simply being of opposite sign. The closure phase is then determined simply by adding the phases at three independent points with the correct sign.

It is clear from Fig. 5(b) that any redundant interferometer spacings would lead to superposed amplitudes and phases, thus introducing an undesirable noise component as discussed in Sec. III $c$.

The full width at half-maximum of the intensity (i.e., the seeing) in the example shown here was 1.0 arcsec, and the integration time was $0.1 \mathrm{~s}$. In all the observations reported here, the object, $\alpha$ Lyr (Vega), was observed near the zenith, the typical airmass being 1.01. Thus the atmospheric refractive effects were small. In order to observe objects at greater airmasses, an atmospheric-dispersion corrector must be used.

Integration times in the range $\tau=0.01-40 \mathrm{~s}$ were tried. Detectable fringes were observed for all $\tau$, but the visibility amplitudes were significantly below unity in all of our observations. We corrected the visibility amplitudes for the effects of refraction using the model discussed by Filippenko (1982). The typical correction was about $20 \%$ and the resulting visibility amplitudes for different integration times are shown in Fig. 6(a). We have simulated the atmospheric effects on the visibility amplitude (see the Appendix). The expected variation of visibility with integration time based on a simple model for the atmospheric turbulence is also shown in Fig. 6(a). The fit to the simulated curves is fair, especially considering the simplicity of our model and the fact that the visibility amplitudes are so difficult to calibrate.

The closure phases were also measured for different integration times, and the rms scatter in the observed closure phase as a function of integration time is shown in Fig. 6(b). The curves indicate the expected noise in closure-phase measurements, again based on our simple atmospheric model (see the Appendix). The expected rms scatter for closure phases randomly distributed between $-180^{\circ}$ and $+180^{\circ}$ is $104^{\circ}$, the value seen for long integration times. It is clear, however, that in conditions of 1 arcsec seeing the closure phase can be determined from observations with $\tau \sim 0.1 \mathrm{~s}$. The agreement of the observed scatter with the expected value for long integration times is rather better than expected based on the errors.

The results of these tests support the finding of Baldwin et al. (1986) that there are no unforeseen difficulties associated with the measurement of closure phase by this method.

\section{THE HYBRID MAPPING TECHNIQUE IN OPTICAL INTERFEROMETRY}

The problem of constructing an image from the amplitudes and bispectra can be reduced to a form that is identical to the problem of constructing an image from amplitudes and closure phases in radio astronomy. The methods employed for constructing images in radio astronomy from partially calibrated and uncalibrated observations, commonly known as hybrid mapping or self-calibration, have been reviewed by Pearson and Readhead (1984) and Readhead

\section{(C) American Astronomical Society - Provided by the NASA Astrophysics Data System}



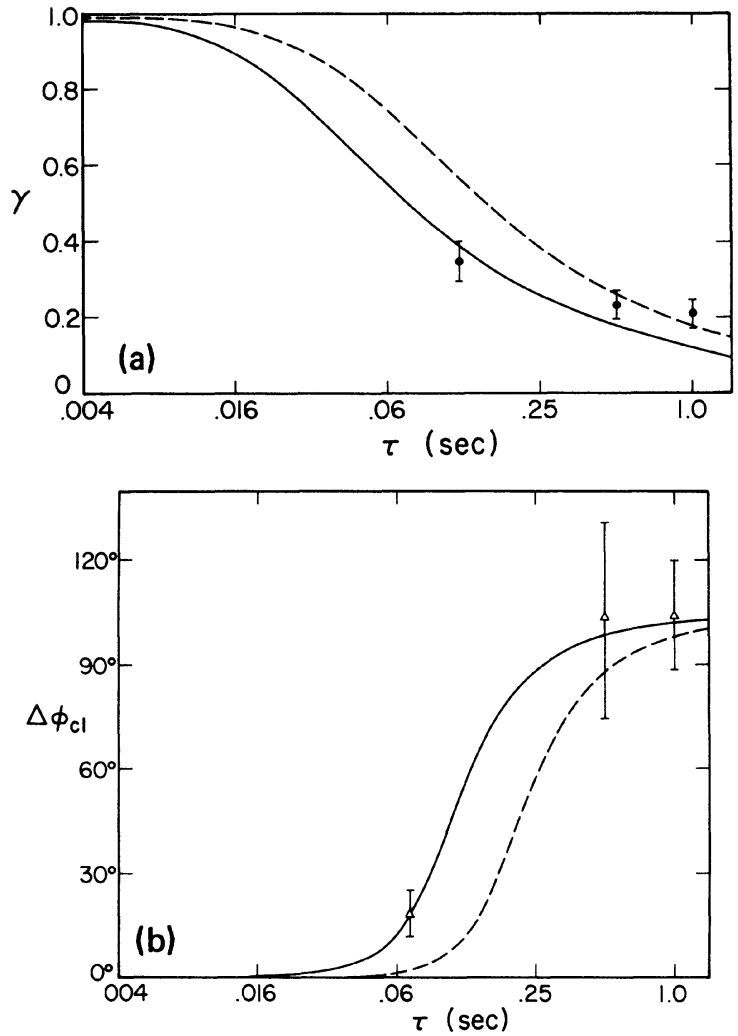

FIG. 6. Comparison of observed parameters of the visibility function ( $\pm 1 \sigma$ error bars) with values determined from the simple atmospheric model described in the Appendix. (a) The visibility amplitude and (b) the rms scatter in the closure phases are shown for different integration times $\tau$ and for conditions of $0.5 \operatorname{arcsec}$ seeing (dashed curve) and 1 arcsec seeing (solid curve). The agreement between the observations and theory has been optimized by adjusting the wind speed. In this example, a wind speed of $2 \mathrm{~m} / \mathrm{s}$ has been assumed.

(1988). The adaptation and application of these methods to the optical case is straightforward.

\section{a) Imaging Simulations}

In order to demonstrate the potential for making optical images using nonredundant masks, we have carried out a number of simulations. We chose to make blind tests to avoid making unjustified assumptions about the objects in the image-construction procedure. The original models were invented by one of us and not revealed to two of us until we had produced the final image. These simulations are realistic in the following sense:

(1) They have $(u, v)$ coverage that is comparable to that which could be realized in practice with ten-hole masks. The mask was rotated through $180^{\circ}$ in steps of 1.25 to increase the aperture coverage and simulate real observations.

(2) the typical noise in the closure-phase measurements is in the range $5 \%-20 \%$, i.e., these are the noise levels that we must achieve if we are to make images with dynamic ranges of $\sim 100$. Since we are considering only bright objects here, we expect that comparable signal-to-noise ratios could easily be obtained with a few hundred independent exposures, assuming that the noise in the closure phase in each exposure is about 1 radian.
1) Tests with visibility amplitudes and closure phases

There is now considerable experience in the construction of images from the visibility amplitudes and closure phases, since this method is the basis of all but a few VLBI maps. We first carried out four blind tests using visibility amplitudes and closure phases. The procedure we used was identical to the self-calibration commonly used in VLBI (see, for example, Cornwell and Wilkinson 1981; Pearson and Readhead 1984).

A typical example is shown in Fig. 7. In Fig. 7(a) is shown
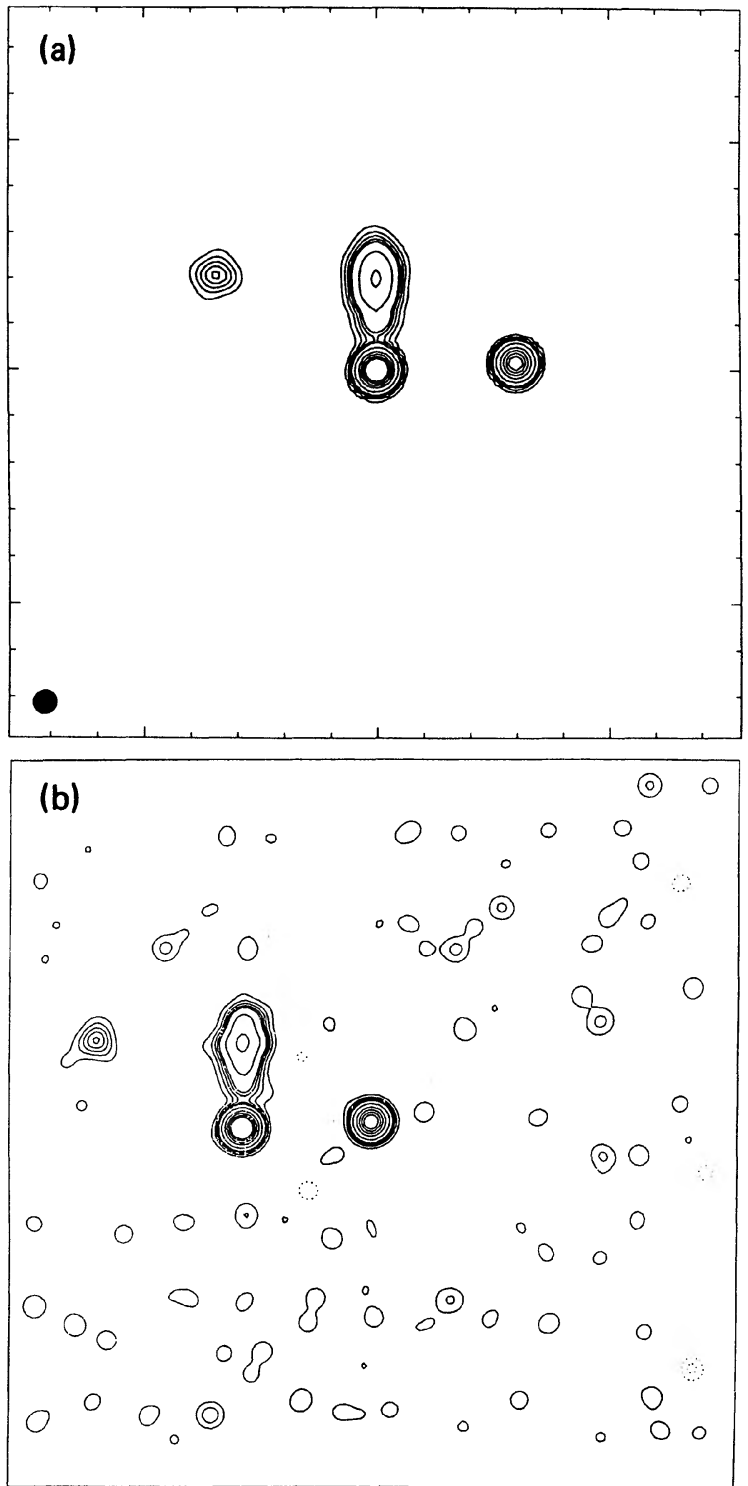

Fig. 7. Blind test construction of object from simulated observations with a ten-hole mask. In this test, well-calibrated amplitudes were assumed, and closure phases were used. Random noise of $5 \%-20 \%$ has been added to the amplitudes and closure phases. (a) Image constructed from full amplitude and phase data; (b) blind test construction from amplitudes and closure phases after ten iterations. The contour levels in both images are at $-1 \%, 1 \%, 2 \%, 3 \%, 4 \%, 5 \%, 10 \%, 20 \%, 30 \%$, $40 \%, 50 \%$ of the peak brightness. Note that a dynamic range, defined as the ratio of the peak brightness to the rms brightness in a blank part of sky, of 150:1 has been achieved. The hatched circle at the lower left indicates the size of the beam (FWHM $=0.026$ arcsec). 
a construction of the original model from a complete set of the interferometry data; i.e., using all the visibility amplitudes and visibility phases. Thus for each observation with ten stations there were 45 amplitudes and 45 phases. The image obtained in our blind test after ten iterations is shown in Fig. 7(b). This image was based on the visibility amplitudes and closure phases; thus each observation consisted of 45 amplitudes and 36 independent closure phases. The selfcalibration method, which relies on the positivity constraint on the brightness distribution, has enabled us to derive all the visibility phases.

A more complicated example is shown in Fig. 8. The level
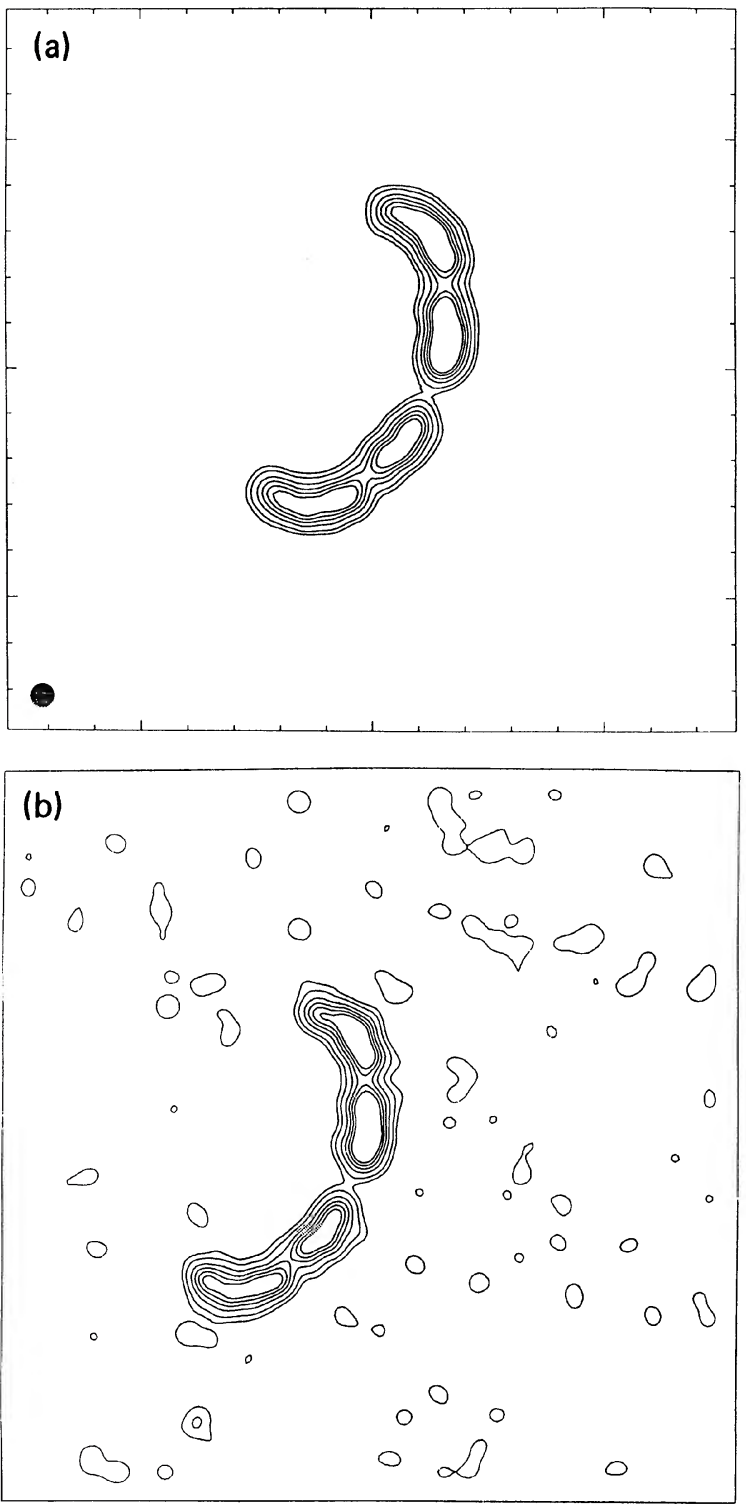

Fig. 8. Blind test of a very extended object with a ten-hole mask. The object is 20 beamwidths long. In this test, well-calibrated amplitudes were assumed and closure phases were used. Random noise of 5\%-20\% was added to the amplitudes and closure phases. (a) Image constructed from full amplitude and phase data; (b) blind test constructed from amplitudes and closure phases after ten iterations. The contour levels are $5 \%, 10 \%, 20 \%, 30 \%, 40 \%, 50 \%$ of the peak brightness. The dynamic range is about 30 . The hatched circle at the lower left indicates the size of the beam (FWHM $=0.026$ arcsec). of errors is considerably higher than the random-noise level, so it is clear that there remain some systematic effects which have not been eliminated by the self-calibration procedure.

\section{2) Tests with phases alone}

There is a significant difference between the phase and amplitude effects: All of the phase effects, except the geometric effects, are random and introduce no systematic bias, but all of the amplitude problems discussed in Sec. II reduce the visibility amplitude. Furthermore, the reduction in amplitude is generally greater on longer baselines. Thus the visibility amplitudes may be very hard to calibrate, and even the use of closure amplitudes may not eliminate many of these errors. Ironically, the phases, which are impossible to calibrate, may well prove more useful, via the closure phase, in the construction of optical images than the amplitudes, which can be calibrated to some degree.

The relative importance of phase and amplitude information in the construction of images depends on a number of factors including the signal-to-noise ratio, the complexity of the image, and the coverage of the aperture plane. Baldwin and Warner (1976) showed that it is possible in some circumstances to construct images from the visibility amplitudes alone; i.e., with only half the information. In some instances it is possible to construct images from the visibility phases alone, and Oppenheim and Lim (1981) have shown that if the visibility amplitudes from one image are combined with the visibility phases from another image, then the resulting image in some cases resembles closely the image from which the phase has been taken. This is by no means always the case, however. In particular, this procedure fails for some very simple images consisting of only a few $(\sim 4)$ isolated components. We have repeated the Oppenheim and Lim procedure on images consisting of a small number of isolated simple sources, and find that sometimes the resulting image bears no more relation to the original image from which the phase is taken than to that from which the amplitude is taken. Nevertheless, we have found that it is possible to construct the image correctly even in these cases if the amplitude information is ignored completely (i.e., if the closure amplitudes are permitted to change from one iteration to the next).

\section{3) Tests with closure phases alone}

The development of image-construction procedures that rely chiefly on the determination of closure phases may be important in the case of optical interferometry since, as discussed above, there are a number of effects that can corrupt the closure amplitudes.

We were therefore motivated to push the hybrid mapping technique even further, to see if images could be constructed from the closure phases alone. We have had some success in VLBI in the construction of images from closure phases without amplitude information. The maps of 3C 84 and 3C 345 published by Readhead et al. (1983) were derived from models based on closure phases alone, since amplitude calibration was so difficult at that time at $22 \mathrm{GHz}$. After deriving fits to the closure phases, the closure amplitudes were added to make the final maps.

We therefore carried out a further set of blind tests to explore the potential for making images based on the closure phases alone.

The results, although preliminary, are very encouraging. One of our blind tests is shown in Fig. 9. In Fig. 9(a) is 


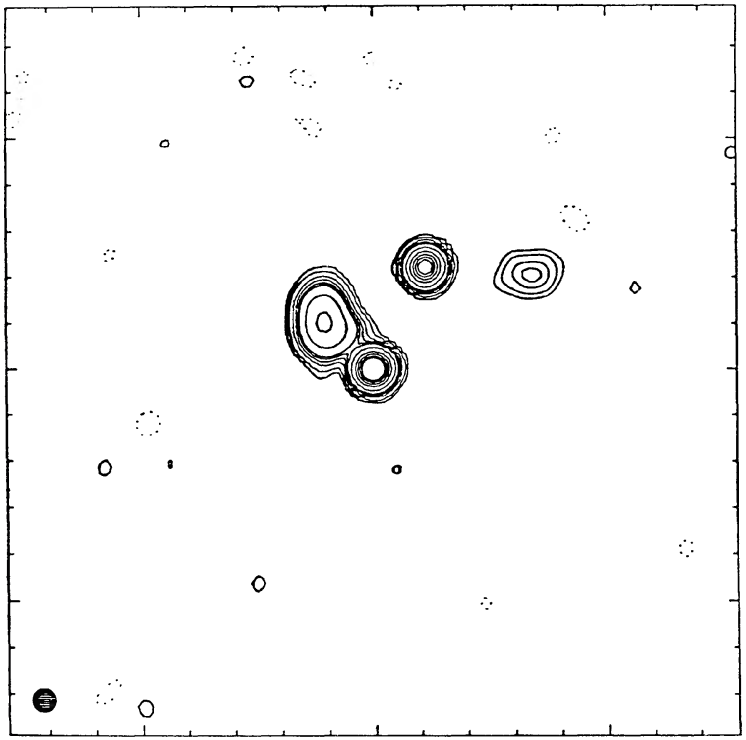

(a)

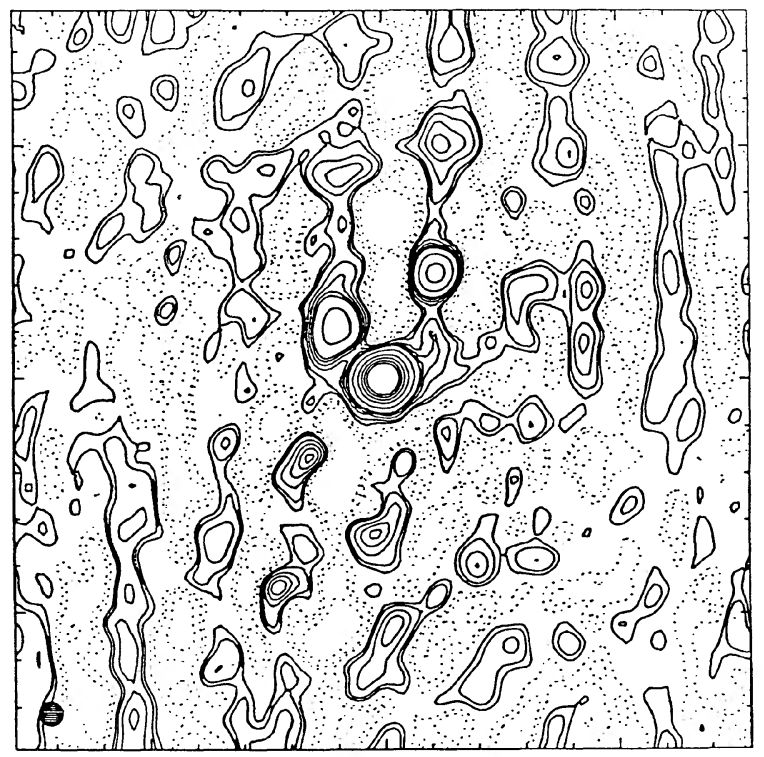

(b)

shown the image constructed from all the visibility datai.e., each observation consists of 45 amplitudes and 45 phases. In Fig. 9(c) is shown the result of our blind test after ten iterations using only the closure phase-i.e., each observation consisted of 36 closure phases and no amplitude information. The procedure we have used is as follows: We use, as a starting model to provide amplitudes, a single point source in the center of the field. We then apply the usual self-calibration technique of adjusting phases and amplitudes of the model consistent with the closure phases, Fourier transform the data to produce a 'dirty map' and CLEAN (see, for example, Pearson and Readhead 1984) the dirty map, thus producing a new model of the source. We then take the amplitudes of this model, together with the closure phases, and perform the second iteration. The procedure is repeated until the map has converged to a stable image. The result of the first iteration, for which the input amplitudes were all unity

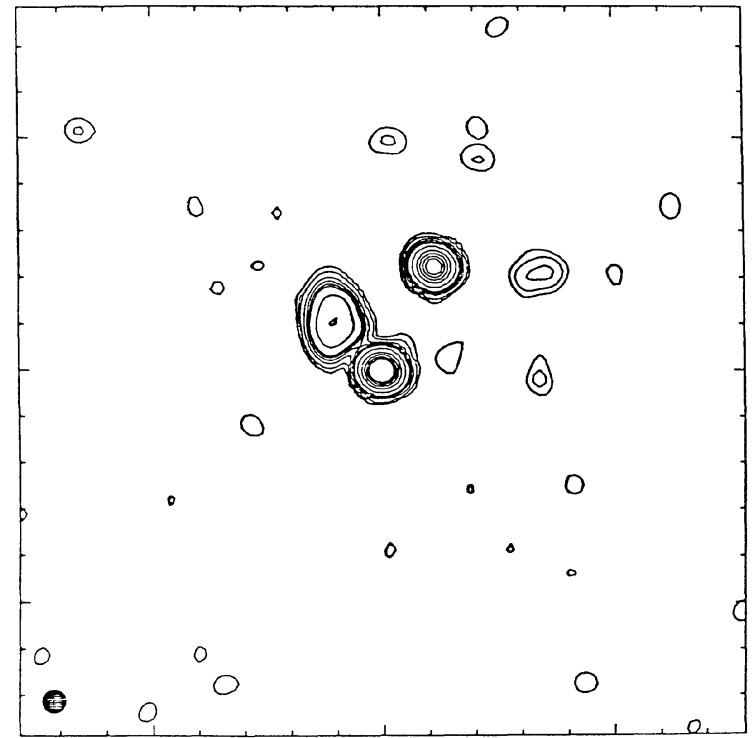

(c)

FIG. 9. Blind test using closure phases alone with ten-hole mask. In this test, the initial starting model was a single point source at the center of the field. No amplitude information was used. Thus the initially assumed amplitudes were all unity. Random noise of 5\%-20\% was added to the closure phases. (a) The image constructed from the full amplitude and phase data that would be obtained with a perfectly coherent ten-aperture array. (b) The blind-test image constructed after one iteration; i.e., the image obtained from the closure phases and assuming unit amplitude. (c) The blind-test image constructed after ten iterations. In each iteration, the closure amplitudes from the image produced in the previous iteration were used together with the 'observed' closure phases. The contour levels in all images are $-2 \%,-1 \%,-0.5 \%, 0.5 \%, 1 \%$, $2 \%, 3 \%, 4 \%, 5 \%, 10 \%, 20 \%, 30 \%, 40 \%, 50 \%$ of the peak brightness. Note that the noise level in the image derived from the closure phases alone is about twice that of the image with the perfectly coherent array. This is to be expected since only $36 / 90$ of the information was used. Note also that a dynamic range of over 200 has been achieved. The hatched circle at the lower left indicates the size of the beam (FWHM $=0.026$ arcsec). since the model at that stage was a point source, is shown in Fig. 9(b).

Comparison of Fig. 9(c) with Fig. 9(a) shows that the noise level in the map made from closure phases alone is about a factor of 2 greater than in the map made from fullvisibility data. This is to be expected, as only $36 / 90$ of the data was used. We would therefore expect the noise to be about a factor of 1.6 greater in Fig. 9(c). In practice, we have not quite achieved this level because there are some systematic effects which have not been completely eliminated in the self-calibration procedure.

We are not suggesting that any arbitrary image can be constructed from high-signal-to-noise-ratio closure-phase observations alone with a ten-hole mask. Rather, these simulations demonstrate the complexity of objects that could easily be constructed from such observations. For many important astrophysical observations, the basic images would 
be quite simple - e.g., double sources, small groups of stars, stellar features, etc. All of these would have a fairly good starting model, and reliable images could be recovered from the closure phases with a minimum of amplitude information. Likewise, active nuclei might well have a strong, unresolved optical core, which would serve as an ideal phase reference and make a point source a good starting model.

More work is needed in this area to determine the range of source complexity that is recoverable from closure-phase observations alone.

\section{CONCLUSIONS}

We have shown that it is possible to measure the closure phase at optical wavelengths on the $200 \mathrm{in}$. telescope, and that observations of the closure phase alone can be used to construct diffraction-limited images using software originally developed for VLBI. We have also estimated the degree of corruption of the closure phases by the redundant baselines in full apertures, and hence shown that the nonredundantmask technique is superior to the use of full apertures for observations of objects brighter than 8 mag. This is a conservative estimate, and we expect the nonredundant mask to be superior to the fully filled aperture method for objects significantly fainter than this, especially under good seeing conditions. We find, further, that the noise observed in the closure phases is consistent with that predicted from simple atmospheric theory. It appears that the only substantive issue in the construction of diffraction-limited optical images from the ground is that of sensitivity. Provided that the objects are bright enough, standard radio astronomy techniques can be used and very high-dynamic-range images can be constructed.

We thank T. J. Cornwell, S. Kulkarni, and P. N. Wilkinson for useful discussions, and the staffs at Palomar Observatory and the Owens Valley Radio Observatory for their assistance. We also thank the referee for constructive comments and suggestions. This work was supported in part by grants AST85-09822 and AST86-13059 from the National Science Foundation and by a grant from the W. M. Keck Foundation.

\section{APPENDIX: APPLICATION OF ATMOSPHERIC SEEING THEORY TO CLOSURE PHASE}

The details of the propagation of electromagnetic waves through a medium with random variations in refractive index depend on the spatial spectrum of the irregularities. Much effort has been expended determining the temporal and spatial spectra of the refractive-index irregularities in the atmosphere (Coulman 1985). For the purposes of this discussion we will assume that the atmospheric-turbulence energy spectrum follows Kolmogorov's $11 / 3$ power law (Kolmogorov 1941), and that the temperature, humidity, and refractive-index inhomogeneities are caused by turbulence for which the corresponding refractive-index spectrum $\Phi_{n}(\kappa)$ is given by (e.g., Tatarski 1961)

$$
\Phi_{n}(\kappa)=0.033 C_{n}^{2} \kappa^{-11 / 3} \exp \left(-\kappa^{2} / \kappa_{m}^{2}\right),
$$

where $l_{0} \equiv 2 \pi / \kappa_{m}$ is the inner scale of the turbulence. This expression holds provided $\kappa>\kappa_{0}$, where $L_{0}=2 \pi / \kappa_{0}$ is the outer scale of turbulence. Denoting the propagation path length through the inhomogeneous medium by $x_{1}$, the structure constant of refractive index $C_{n}^{2}(x)$, which determines the amount of scattering as a function of $x$, is related to the seeing by

$$
\int_{0}^{x_{1}} C_{n}^{2}(x) d x=4.4 \times 10^{-13} \Theta^{5 / 3}\left(m^{1 / 3}\right),
$$

where $\Theta$ is the seeing in arcsec and $x$ is measured in meters (C. Roddier 1981; F. Roddier 1981). We will refer to this quantity as the 'scattering strength' of the atmosphere.

We now consider interferometry with a nonredundant mask on a large telescope. The mask transmits light from small patches on the primary mirror. In order to apply conventional Michelson interferometry, each patch must be coherently illuminated; i.e., the diameter $a_{0}$ cannot be much greater than $r_{\mathrm{coh}}$. The determination of just how much greater it can be, while still yielding usable fringes, is one of the main objectives of our preliminary studies on the 200 in. telescope. As the patch size is increased beyond $r_{\text {coh }}$, the image breaks up into speckles, whose average size is set by the diffraction limit of the patch, which are spread over a seeing disk of diameter determined by $r_{\text {coh }}$.

The theoretical determination of the fringe phase and amplitude when $a_{0}>r_{\text {coh }}$ is complicated (Rhodes and Goodman 1973), and we do not consider it further here. As long as the intensity pattern has not broken up into individual speckles, the amplitude and phase of the fringes will be little altered by the finite size of the patches. In this case the patches can be approximated by points. The temporal power spectrum of the phase difference measured at two points separated by a distance $\rho, w_{\delta \phi}(f)$, can be determined (Tatarski 1967; Lawrence and Strohbehn 1970):

$$
\begin{aligned}
w_{\delta \phi}(f)= & 0.066(2 \pi / \lambda)^{2} v^{5 / 3} f^{-8 / 3} \\
& \times \int_{0}^{x_{1}} C_{n}^{2}(x) d x, \text { for } \rho \gg\left(\lambda x_{1}\right)^{1 / 2},
\end{aligned}
$$

where we assume Taylor's hypothesis of frozen-in turbulence (1935), and $v$ is the average wind-velocity component perpendicular to the direction of propagation of the light.

Colavita and Shao (1987) have measured $w_{\delta \phi}(f)$ under 0.5 arcsec seeing conditions. They observe the $f^{-8 / 3}$ behavior and measure a power of $1.2 \times 10^{-3} \mu \mathrm{m}^{2} / \mathrm{Hz}$ at $f=10 \mathrm{~Hz}$. This value is in agreement with the value for the scattering strength given above if we assume that the wind velocity was $12 \mathrm{~m} / \mathrm{s}$. The wind speed at the time of our observations was much lower than this, and we find that our observations agree with the simple theory if we assume a wind speed of 2 $\mathrm{m} / \mathrm{s}$ (see below).

The temporal power spectrum of phase fluctuations at one point, $w_{\phi}(f)$, is one-half the value given by Eq. (A1) (Lawrence and Strohbehn 1970). We have used this in a simulation to calculate random-phase changes caused by Kolmogorov turbulence along the line of sight to four patches on the primary mirror with separation $\gg r_{\text {coh }}$. These phase changes are then used to calculate the effects caused by loss of temporal coherence on the visibility amplitude, the closure phase, and the closure amplitude. These effects have been estimated from the spectrum given by Eq. (A1) by the following procedure. We first generate a time series of random-phase variations with a white-noise spectrum. This series is then Fourier transformed and multiplied by a power spectrum with power law index $-8 / 3$, and with the appropriate normalization given by Eq. (A1). Transforming back to the time domain then yields random-phase fluctuations with the desired spectral characteristics.

The phases at time $t$ are calculated at four apertures 1,2,3 and 4 and the resultant complex visibilities $\Gamma(\tau)$ are calculated by vector addition of the visibilities in successive $1 \mathrm{~ms}$ 
intervals up to the integration time $\tau$. The amplitude of the fringes is $\gamma=|\Gamma|$ and the phase is $\arg (\Gamma)$. The closure phases and closure amplitudes are then calculated from these values (cf. Readhead et al. 1980). We estimate the effect of our finite observing bandwidth (1000 $\AA$ ) on the visibility amplitudes to be less than $3 \%$, and we have therefore ignored this effect in our simulations.

In Fig. 10(a) we show the individual phases for three

(a)

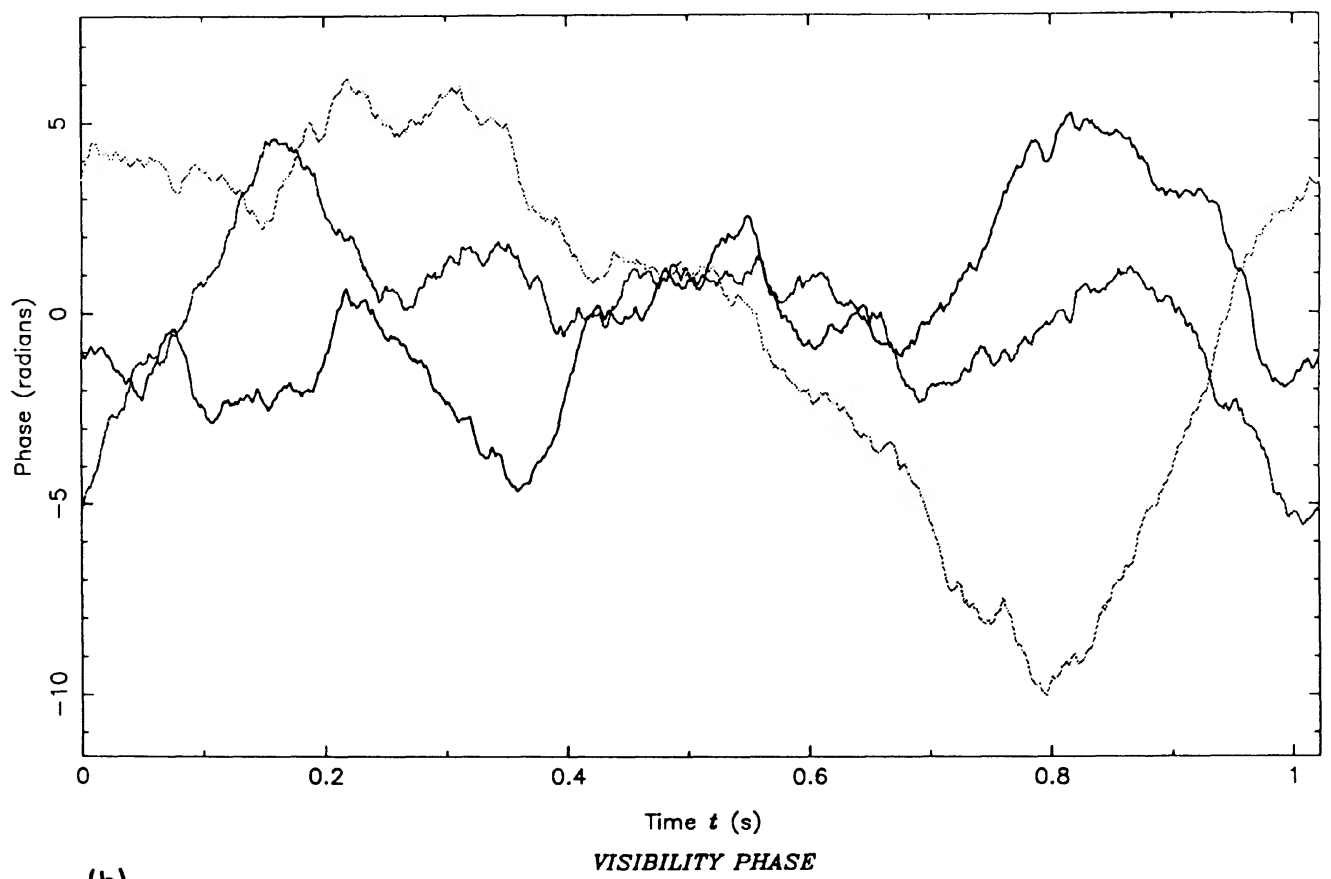

(b)

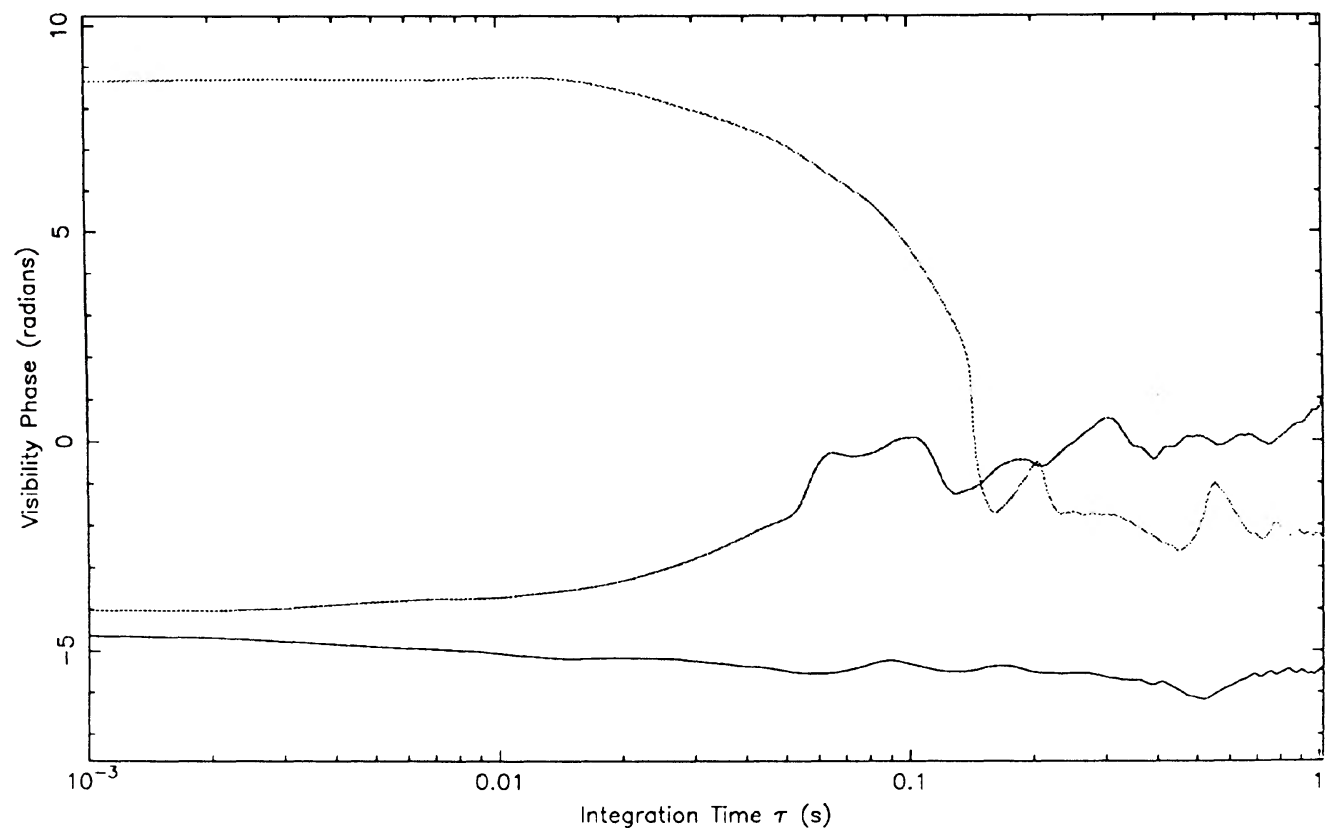

FIG. 10. (a) Example of phases observed at three apertures calculated for the simple model of Kolmogorov turbulence described in the Appendix. (b) Visibility phases observed on the three baselines. Note that the phases show large offsets, even for short integration times, due to the phase variations imposed by the atmosphere. Note also that the phases on individual baselines vary by more than a radian for times longer than $0.03 \mathrm{~s}$. (c) Visibility amplitudes observed on the three baselines. Note the large variation in amplitudes between the different baselines. Note also that the amplitudes on two of the baselines drop below 0.5 for integration times longer than $0.04 \mathrm{~s}$. (d) Closure phase observed on the closed loop of three baselines. Note that the closure phase varies by less than 0.06 radians for integration times up to $0.03 \mathrm{~s}$, and by less than 1 radian up to an integration time of 0.1 s. The zero value of the closure phase at short integration times illustrates the normal cancellation of propagation errors for intervals shorter than the coherence time. The small variations of the closure phase for times significantly longer than $\tau_{\text {coh }}$ illustrate the partial cancellation of redundancy-noise terms (see the text). 
(c)

VISIBILITY AMPLITUDE



(d)

CLOSURE PHASE

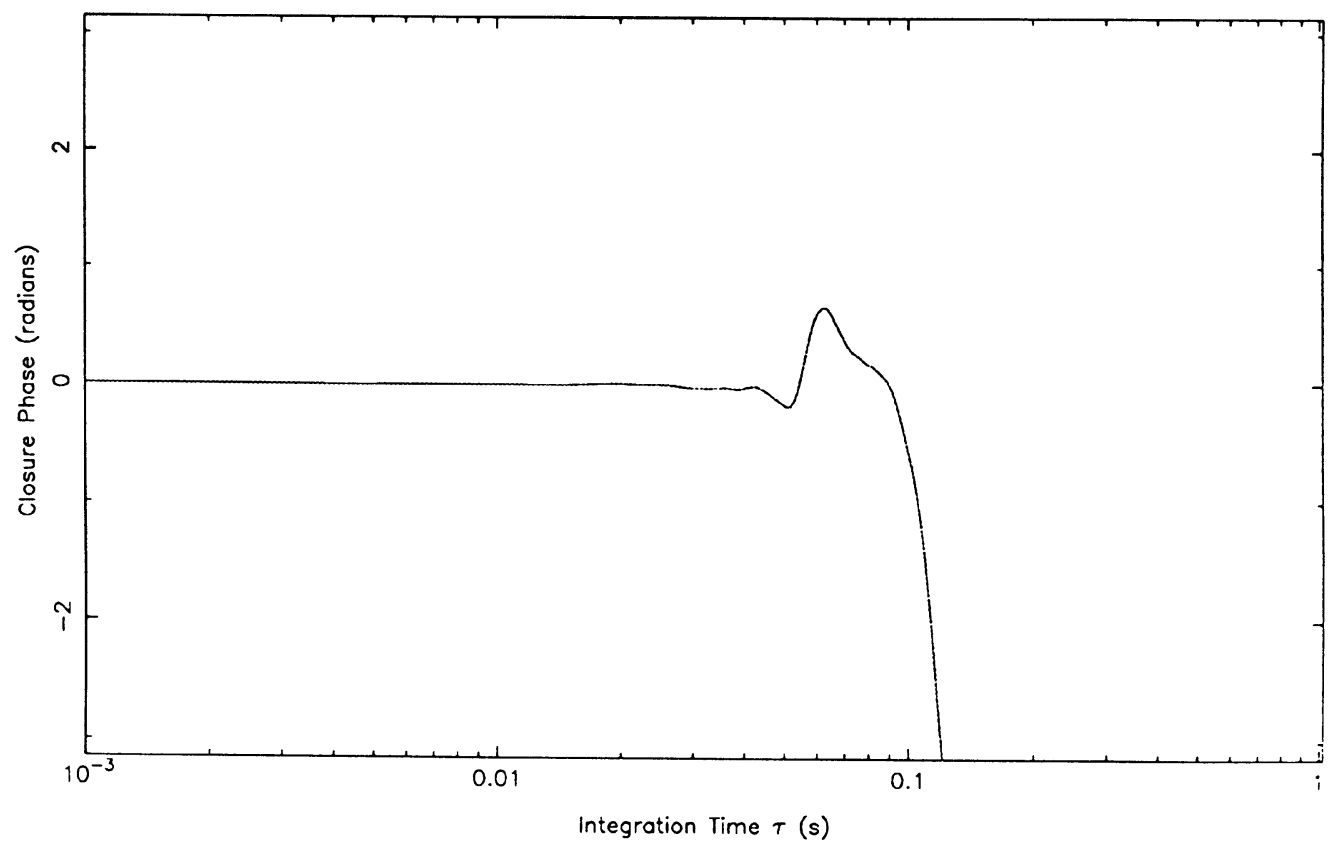

FIG. 10 (continued)

patches from a simulation in which we calculate random phases due to the atmosphere, by the method outlined above, over a period of $1 \mathrm{~s}$. We assume 1 arcsec seeing and a wind speed of $2 \mathrm{~m} / \mathrm{s}$. Note that the phase variations caused by the atmosphere amount to many radians. In this simulation, we have made the simplifying assumption that the phase variations are uncorrelated at the different patches, and since the variations will be correlated on scales larger than the separation of the patches, we have not included contributions from scales larger than the patch separation. The average power spectrum at four apertures in one of our simulations is compared with the observations by Colavita and Shao (1987) in Fig. 11.

In Fig. 10(b) we also show the visibility phases as a function of integration time on three baselines. Note that the visibility phases, which would be zero for a point source observed in the absence of the atmosphere, amount to many radians. The visibility amplitude is shown in Fig. 10(c), and 


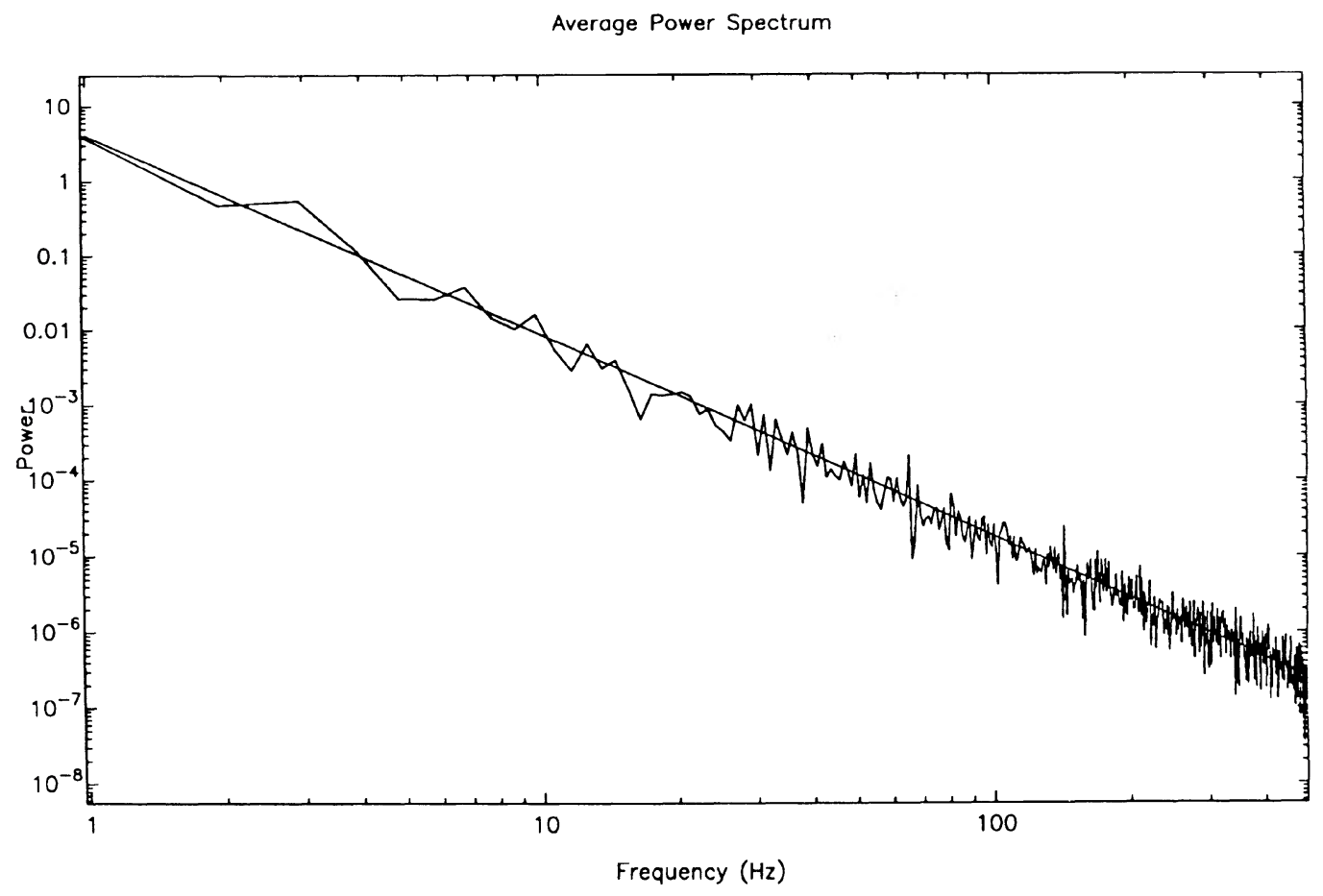

FIG. 11. Comparison of simulated spectrum with observations by Colavita and Shao (see the Appendix). The straight line is a fit to the observations of Colavita and Shao, normalized for a wind speed of $2 \mathrm{~m} / \mathrm{s}$, and allowing for the fact that these are singleaperture spectra. The noisy spectrum is the average of four spectra generated in our simple atmospheric simulation. Three of the spectra used here are shown in Fig. 10(a).

Fig. 10(d) shows the closure phase.

There are a number of features in the results of these simulations that are not unexpected, but are nevertheless worth pointing out:

(1) The instantaneous phase fluctuations introduced by the atmosphere (Fig. 10(a)) are so large that the phases on individual interferometer baselines (Fig. 10(b)) are meaningless. This accounts for Hanbury Brown's statement (1968) that 'it is not practicable to observe the phase of the fringe pattern at optical wavelengths.' Furthermore, significant variations, in addition to the original offset, occur over timescales longer than $30 \mathrm{~ms}$, showing that observations of duration longer than 30 ms will show the effects of loss of coherence; i.e., the amplitude will be reduced - as is indeed the case.

(2) The visibility amplitudes begin to depart significantly, i.e., by more than $5 \%$, from unity at timescales longer than 10 ms. Thus, with 1 arcsec seeing, calibration of the amplitudes is difficult for observations of duration longer than 10 ms.

(3) The closure phase is well behaved, and deviates by less than 1 radian up to integration times of $100 \mathrm{~ms}$. Note that the individual phases are meaningless, no matter how short the integration time, because of random-phase differences along the lines of sight to the two apertures, but the closure phase is well behaved for short integrations. This makes possible the extraction of structural information about the object through the closure phase. This is a graphic demonstration of the cancellation of systematic phase errors in the closure phase. Note also that the individual visibility phases begin to depart from the original values by more than a radian for an integration time of around $30 \mathrm{~ms}$, whereas the closure phase remains within 1 radian for an integration time of $100 \mathrm{~ms}$; i.e., this example illustrates the point that measurements of the closure phase can be made with integration times about a factor of 2 longer than $\tau_{\text {coh }}$ due to the cancellation of redundancy terms of the second kind (see Sec. III $c$ ). A similar result holds for the closure amplitudes. Most of the atmospheric effects cancel to high precision in the closure phase and closure amplitude. Only when the visibility amplitudes drop below 0.5 and the phase variations within an integration rise to about 3 radians do the closure phases and amplitudes begin to become seriously corrupted. Perhaps it is worth pointing out again here that even when this information is badly affected by noise; for example, through the use of integration times much longer than the coherence time, the closure phase term in the bispectrum will still add coherently, so that the signal-to-noise ratio will still increase in proportion to $V N$ if we have $N$ exposures (see Sec. III $c$ ). Similarly, apertures larger than the lateral coherence length can be used ( $\mathrm{cf}$. our use of $20 \mathrm{~cm}$ apertures under conditions of 1 arcsec seeing), and large bandwidths can be used.

We have simulated the effects of the loss of temporal coherence as a function of $\tau$ under different seeing conditions, by calculating a series of random phases, such as those shown in Fig. 10(a), at four apertures, and then computing visibility amplitudes, closure phases, and closure amplitudes as a function of integration time for each simulation. From the statistics of these simulations we calculate the statistical behavior of the relevant parameters. The effects on visibility amplitude and closure phase are shown in Figs. 6(a) and $6(\mathrm{~b})$, respectively, in Sec. IV. It can be seen that with 1 
arcsec seeing closure phases can be measured with $\tau \sim 0.1 \mathrm{~s}$, but that for integration times longer than $0.1 \mathrm{~s}$ the random fluctuations in the closure phase due to the loss of coherence will greatly increase the noise in the closure-phase measurements, in good agreement with the observations. The vector addition of these phases, as in the bispectrum, preserves the closure-phase information, and enables us to extract this even from very noisy data (Lohmann et al. 1983; Rogers et al. 1984).

The results of these simulations demonstrate that we can measure closure phases, even when the visibility has dropped significantly below unity due to the loss of temporal coherence. Our observations and those of Baldwin et al. (1986) confirm this finding.
Baldwin, J. E., Haniff, C. A.,Mackay, C. D., and Warner, P. J. (1986). Nature 320, 595.

Baldwin, J. E., and Warner, P. J. (1976). Mon. Not. R. Astron. Soc. 175, 345.

Brown, T. M. (1978). J. Opt. Soc. Am. 68, 883.

Colavita, M. M., and Shao, M. (1987). Proceedings of the ESO NOAO Workshop on High Angular Resolution Astronomy from the Ground, held in Oracle, Arizona, 12-15 January 1987, edited by J. Goad (NOAO, Tucson).

Cornwell, T. J. (1987). Astron. Astrophys. 180, 269.

Cornwell, T. J., and Wilkinson, P. N. (1981). Mon. Not. R. Astron. Soc. 196, 1067.

Coulman, C. E. (1985). Annu. Rev. Astron. Astrophys. 23, 19.

Currie, D. G., Knapp, S. L., and Liewer, K. M. (1974). Astrophys. J. 187, 131.

Filippenko, A. V. (1982). Publ. Astron. Soc. Pac. 94, 715.

Fienup, J. R. (1978). Opt. Lett. 3, 27.

Fienup, J. R. (1984). In Indirect Imaging, edited by J. A. Roberts (Cambridge University, Cambridge).

Hanbury Brown, R. (1968). Annu. Rev. Astron. Astrophys. 6, 13.

Hanbury Brown, R., Davis, J., Herbison-Evans, D., and Allen, L. R. (1970). Mon. Not. R. Astron. Soc. 148, 103.

Haniff, C. A., Mackay, C. D., Titterington, D. J., Sivia, D., Baldwin, J. E., and Warner, P. J. (1987). Nature 328, 694

Hofmann, K.-H., and Weigelt, G. (1986). Astron. Astrophys. 167, L15.

Jennison, R. C. (1953). Ph.D. thesis, University of Manchester.

Jennison, R. C. (1958). Mon. Not. R. Astron. Soc. 118, 276.

Knox, K.T., and Thomson, B. J. (1974). Astrophys. J. Lett. 193, L45.

Kolmogorov, A.N. (1941). In Turbulence, translated by S. K. Friedlander and L. Topper, 1961 (Interscience, New York).

Labeyrie, A. (1970). Astron. Astrophys. 6, 85.

Lawrence, R. S., and Strohbehn, J. W. (1970). Proc. IEEE 58, 1523.

Liu, C. Y. C., and Lohmann A. W. (1973). Opt. Commun. 8, 372.

Lohmann, A. W., Weigelt, G., and Wirnitzer, B. (1983). Appl. Opt. 22, 4028 .

McAlister, H. A. (1985). Annu. Rev. Astron. Astrophys. 23, 59.

Michelson, A. A. (1920). Astrophys. J. 51, 257.

Michelson, A. A., and Pease, F. G. (1921). Astrophys. J. 53, 249

Oppenheim, A. V., and Lim, J. S. (1981). Proc. IEEE 69, 529.

Pearson, T. J., and Readhead, A. C. S. (1984). Annu. Rev. Astron. Astrophys. 22, 97.
Peebles, P. J. E. (1980). The Large Scale Structure of the Universe (Princeton University, Princeton, NJ).

Readhead, A. C. S. (1988). In Proceedings of a Workshop on the Impact of Instrumentation on Astronomy, commemorating the 70th birthday of $\mathbf{R}$. Hanbury Brown, held in Herstmonceux, 24-26 September 1986, edited by J. V. Wall (Cambridge University, Cambridge) (in press).

Readhead, A. C. S., Hough, D. H., Ewing, M. S., Walker, R. C., and Romney, J. D. (1983). Astrophys. J. 265, 107.

Readhead, A. C. S., Walker, R. C., Pearson, T. J., and Cohen, M. H. (1980). Nature 285, 137.

Readhead, A. C. S., and Wilkinson, P. N. (1978). Astrophys. J. 223, 25

Rhodes, W. T., and Goodman, J. W. (1973). J. Opt. Soc. Am. 63, 647.

Roddier, C. (1981). In Progress in Optics, Vol. XIX, edited by E. Wolf (North Holland, Amsterdam), p. 284.

Roddier, C., and Roddier, F. (1987). Proceedings of An International Conference on the Identification, Optimization and Protection of Optical Telescope Sites, Flagstaff, May 1986 (NOAO, Tucson).

Roddier, F. (1981). ESO conference on The Scientific Importance of High Angular Resolution at Infrared and Optical Wavelengths, Garching, March 1981, edited by M. H. Ulrich and K. Kjar (ESO, Garching).

Roddier, F. (1987). J. Opt. Soc. Am. 4, 1396.

Rogers, A. E. E., et al. (1974). Astrophys. J. 193, 293.

Rogers, A. E. E., Moffet, A. T., Backer, D. C., and Moran, J. M. (1984). Radio Sci. 19, 1552.

Rogstad, D. H. (1968). Appl. Opt. 7, 585.

Schwab, F. R. (1980). Proc. SPIE 231, 18.

Shao, M., Colavita, M., Staelin, D. H., Johnston, K. J., Simon, R. S., Hughes, J. A., and Hershey, J. L. (1987). Astron. J. 93, 1280.

Shao, M., and Staelin, D. H. (1980). Appl. Opt. 19, 1519.

Tatarski, V.I. (1961). Wave Propagation in a Turbulent Medium, translated by R. S. Silverman, 1961 (McGraw-Hill, New York).

Tatarski, V. I. (1967). The Effects of the Turbulent Atmosphere on Wave Propagation, translated by Israel Program for Scientific Translations, Keter, Jerusalem, 1971 (Nauka, Moscow).

Taylor, G. I. (1935). Republished in Turbulence, edited by S. K. Friedlander and L. Topper (Interscience, New York, 1961), pp. 18-75.

Weigelt, G. P. (1977). Opt. Commun. 21, 55.

Weigelt, G. P. (1978). Appl. Opt. 17, 2660.

Weigelt, G. P., and Wirnitzer, B. (1983). Opt. Lett. 8, 389.

Woolf, N. J. (1982). Annu. Rev. Astron. Astrophys. 20, 367. 